ESAIM: COCV

Vol. 13, No 2, 2007, pp. 305-330

DOI: $10.1051 /$ cocv:2007010
ESAIM: Control, Optimisation and Calculus of Variations

www.edpsciences.org/cocv

\title{
HOMOGENIZATION OF FERROMAGNETIC MULTILAYERS IN THE PRESENCE OF SURFACE ENERGIES*
}

\author{
KÉvin SANTUGINI-RePiQUeT ${ }^{1}$
}

\begin{abstract}
We study the homogenization process of ferromagnetic multilayers in the presence of surface energies: super-exchange, also called interlayer exchange coupling, and surface anisotropy. The two main difficulties are the non-linearity of the Landau-Lifshitz equation and the absence of a good sequence of extension operators for the multilayer geometry. First, we consider the case when surface anisotropy is the dominant term, then the case when the magnitude of the super-exchange interaction is inversely proportional to the interlayer distance. We establish the homogenized equation in these two situations.
\end{abstract}

Mathematics Subject Classification. 35B27, 35K60.

Received February 15, 2005. Revised October 12, 2005 and February 7, 2006.

\section{INTRODUCTION}

Ferromagnetic materials ${ }^{2}$ are defined by several interesting properties. Contrary to paramagnetic or diamagnetic materials, they can have a nonzero magnetization in the absence of any exterior excitation. Their global magnetization presents an hysteresis cycle. The magnetization of a ferromagnet depends not only on the current magnetic field but also on all its previous history. Due to these properties, ferromagnetic materials are now omnipresent in the industry, mainly in four fields of applications: magnetic storage of information, telecommunications, radar protection, and energy management. Therefore, understanding the magnetic behavior of ferromagnets is a prime concern in these fields.

While the basic qualitative properties of ferromagnets have been known since antiquity, it was not until the late forties that quantitative macroscopic models of ferromagnetism were proposed. The micromagnetic model of Brown [5] describes the different energies involved in ferromagnetism and fully characterizes the equilibrium states of the magnetization $\boldsymbol{m}$. Landau and Lifshitz [12] proposed a phenomenological nonlinear PDE to model the evolution in time of the magnetization in ferromagnets,

$$
\frac{\partial \boldsymbol{m}}{\partial t}=-\boldsymbol{m} \wedge \boldsymbol{h}-\alpha \boldsymbol{m} \wedge(\boldsymbol{m} \wedge \boldsymbol{h}),
$$

Keywords and phrases. Ferromagnetism, multilayers, Landau-Lifshitz equation, micromagnetic model.

* Work done while at Paris 13.

${ }^{1}$ UNIGE, Section de mathématiques, Genève, Switzerland; Kevin.Santugini@math.unige.ch

${ }^{2}$ See Aharoni [1], or Trémolet de Lacheisserie [17] for physical aspects of ferromagnetism. 
now known as the Landau-Lifshitz equation. The vector field $\boldsymbol{h}$ depends on $\boldsymbol{m}$ and is given by the micromagnetic model of Brown [5].

Different forms and configurations of ferromagnets can yield very different properties. Ferromagnetic multilayers have been a strong area of research in recent years because they can yield properties, such as giant magnetoresistance, that are difficult to obtain otherwise. See [18], Chapter 20, for a description of the observed properties of ferromagnetic multilayers and their applications. Simply replacing a solid ferromagnetic domain by a ferromagnetic multilayer in the mathematical model would not account for all these interesting properties. Indeed, new physical phenomena in the spacers in between the layers such as super-exchange and surface anisotropy, see [11], must be considered. Super-exchange, penalizes the jump of the magnetization $\boldsymbol{m}$ in between the layers. Surface anisotropy controls the orientation of the magnetization with respect to the normal to the surface in between the different layers. These two phenomena or interactions are modeled in the framework of micromagnetism by adding surface terms to the total energy.

The complexity and the nonlinearity of the equations involved in the micromagnetic model make numerical simulations a necessary tool to correctly predict the magnetic behavior of ferromagnets. Unfortunately, using the micromagnetic model to directly compute the behavior of ferromagnetic multilayers is impractical because the small thickness of each layer would increase the number of cells in the mesh in a prohibitive way. A more practical way is to derive a macroscopic model of the behavior of ferromagnetic multilayers using homogenization techniques. The non-linearity of the equations makes the study of convergence highly nontrivial for multilayered geometries: for such domains, there is no extension operator with "good" properties. The homogenization of ferromagnetic materials has been previously studied by Hamdache [9], and by Haddar and Joly [7] in 2D.

It is the purpose of this paper to homogenize the equations of micromagnetism when the thickness of each layer tends to zero while the thickness of the whole body remains constant. We compute the homogenized equations for different values of the parameters controlling the magnitude of the surface energies with respect to the interlayer distance. In particular, we study three different cases: dominant surface anisotropy with either strong super-exchange - see Theorem 12, or weak super-exchange - see Theorem 14, and very strong super-exchange with weak surface anisotropy — see Theorem 18.

Our contribution is two fold. The first contribution is the correct derivation of the two-scale "limit" of the nonhomogeneous boundary conditions in the weak formulation using two-scale convergence and its variant with periodic surfaces. The second contribution is the correct justification of the limits in nonlinear terms. That is, we show how the presence of surface energies - super-exchange and surface anisotropy - at the interface between the different ferromagnetic layers can add enough rigidity to the system to ensure the necessary compactness for the convergence of nonlinear terms in the weak formulations.

This paper is organized as follows. Section 2 reminds the reader the micromagnetic model of ferromagnetism. Section 3 describes explicitely the multilayer geometry and introduces necessary notations. Section 4 describes the Landau-Lifshitz system in a multilayer configuration to be homogenized as well as the concept of weak solutions to the Landau-Lifshitz system. Section 5 reminds the reader the notion of two-scale convergence. Finally, Section 6 presents the homogenized equations satisfied by the two-scale limit of the weak solution to the Landau-Lifshitz system when the number $N$ of layers tends to infinity in the three different cases.

\section{The Equations of FerRomagnetism}

\subsection{The micromagnetic model}

One model that explains the qualitative properties of ferromagnetism is the micromagnetic model by Brown [5]. We describe it in this section. A more complete description can be found in Halpern and Labbé [8].

\subsubsection{The basic principles}

The magnetic state of a ferromagnet filling domain $\mathcal{O}$ is characterized by its magnetization $\boldsymbol{m}$ : a vector field null outside its body. Inside the ferromagnet, the magnetization has a constant modulus $M_{s}$ equal to 1 after 
scaling, i.e.

$$
|\boldsymbol{m}|= \begin{cases}1 & \text { a.e. in } \mathcal{O} \times \mathbb{R}^{+} \\ 0 & \text { a.e. in } \mathbb{R}^{3} \backslash \mathcal{O} \times \mathbb{R}^{+}\end{cases}
$$

To each interaction $p$ involved in ferromagnetism corresponds an energy $\mathrm{E}_{p}(\boldsymbol{m})$ and an effective magnetic field $\mathcal{H}_{p}(\boldsymbol{m})$ related by the formulae

$$
\begin{aligned}
\mathrm{E}_{p}(\mathbf{0}) & =0 \\
\mathrm{DE}_{p}(\boldsymbol{m}) \cdot \boldsymbol{v} & =-\int_{\mathcal{O}} \mathcal{H}(\boldsymbol{m}) \cdot \boldsymbol{v} \mathrm{d} \boldsymbol{x} \quad \text { for all } \boldsymbol{v} \text { in } \mathrm{H}^{1}\left(\mathcal{O} ; \mathbb{R}^{3}\right) .
\end{aligned}
$$

Let $\mathrm{E}(\boldsymbol{m})$ be the total energy defined as $\mathrm{E}(\boldsymbol{m})=\sum_{p} \mathrm{E}_{p}(\boldsymbol{m})$. The micromagnetic model then defines the equilibrium states as the local minima of $\mathrm{E}$ among vector fields statisfying constraint 2.1.

\subsubsection{The volume energies}

Inside a ferromagnetic domain, three contributions to the total energy are usually considered:

Exchange energy: This energy has the form $\mathrm{E}_{e}(\boldsymbol{m})=\int_{\mathcal{O}} \frac{A(\boldsymbol{x})}{2}|\nabla \boldsymbol{m}|^{2} \mathrm{~d} \boldsymbol{x}$ where $A$ belongs to $\mathcal{C}\left(\overline{\mathcal{O}}, \mathbb{R}_{+}^{*}\right)$. The associated magnetic effective field is $\boldsymbol{h}_{e}=A \Delta \boldsymbol{m}$. Exchange penalizes the variation of microscopic magnetization and allows the existence of a global magnetization.

Anisotropy energy: In ferromagnetic materials, there are often directions of easy magnetization. This is modeled by an energy of the form $\mathrm{E}_{a}(\boldsymbol{m})=\frac{1}{2} \int_{\mathcal{O}}(\mathbf{K} \boldsymbol{m}) \cdot \boldsymbol{m}$, where $\mathbf{K}$ is a continuous field over $\overline{\mathcal{O}}$ into the space of symmetric positive matrices on $\mathbb{R}^{3}$. The associated magnetic effective field is $\boldsymbol{h}_{a}=-\mathbf{K} \boldsymbol{m}$.

Demagnetization field energy: This energy arises from the magnetostatic equations. It is given by $\mathrm{E}_{d}(\boldsymbol{m})=\frac{1}{2} \int_{\mathbb{R}^{3}}\left|\boldsymbol{h}_{d}\right|^{2} \mathrm{~d} \boldsymbol{x}=-\frac{1}{2} \int_{\mathcal{O}} \boldsymbol{m} \cdot \boldsymbol{h}_{d} \mathrm{~d} \boldsymbol{x}$ where the vector field $\boldsymbol{h}_{d}$ is the solution to the magnetostatic system:

$$
\operatorname{rot} \boldsymbol{h}_{d}=0 \quad \text { in } \mathbb{R}^{3}, \quad \operatorname{div}\left(\boldsymbol{h}_{d}+\boldsymbol{m}\right)=0 \quad \text { in } \mathbb{R}^{3},
$$

in the sense of distributions.

Therefore, usually, the total magnetic effective field is defined as

$$
\boldsymbol{h}=A \triangle \boldsymbol{m}-\mathbf{K} \boldsymbol{m}+\boldsymbol{h}_{d}
$$

Let $\mathcal{H}_{d}$ be the linear operator that maps $\boldsymbol{m}$ into $\boldsymbol{h}_{d}$. We have the following theorem:

Theorem 1. The demagnetization field operator $\mathcal{H}_{d}$ maps the space $\mathrm{L}^{2}\left(\mathbb{R}^{3} ; \mathbb{R}^{3}\right)$ into $\mathrm{L}^{2}\left(\mathbb{R}^{3} ; \mathbb{R}^{3}\right)$, is symmetric negative and has a norm smaller than 1 .

Proof. See Friedman [6] for the basic properties of this operator.

\subsubsection{The surface energies}

If a spacer $\mathcal{J}=B \times(-l, l)$ made of weakly magnetic material, is in between a ferromagnetic domain $\mathcal{O}=B \times\left(-L^{-}, L^{+}\right)$physical interactions such as super-exchange and surface anisotropy are present, see Labrune and Miltat [11]. In the micromagnetic framework, these physical interactions are modeled by adding surface energies to the total energy. The super-exchange energy is

$$
\mathrm{E}_{s e}(\boldsymbol{m})=\frac{J_{1}}{2} \int_{B}|\boldsymbol{m}(\hat{\boldsymbol{x}}, l)-\boldsymbol{m}(\hat{\boldsymbol{x}},-l)|^{2} \mathrm{~d} \hat{\boldsymbol{x}}+J_{2} \int_{B}|\boldsymbol{m}(\hat{\boldsymbol{x}}, l) \wedge \boldsymbol{m}(\hat{\boldsymbol{x}},-l)|^{2} \mathrm{~d} \hat{\boldsymbol{x}},
$$


where $J_{1}$ and $J_{2}$ are positive constants. The second term of the super-exchange energy is the biquadratic term. The anisotropic surface energy is

$$
\mathrm{E}_{s e}(\boldsymbol{m})=\frac{K_{S}^{+}}{2} \int_{B \times\{l\}}|\boldsymbol{m}(\boldsymbol{x}) \wedge \boldsymbol{\nu}|^{2} \mathrm{~d} \boldsymbol{x}+\frac{K_{S}^{-}}{2} \int_{B \times\{-l\}}|\boldsymbol{m}(\boldsymbol{x}) \wedge \boldsymbol{\nu}|^{2} \mathrm{~d} \boldsymbol{x}
$$

where $\boldsymbol{\nu}$ is the exterior normal to $B \times\{ \pm l\}$, and $K_{s}^{+}$and $K_{s}^{-}$are two positive real numbers.

Let $\sigma\left(x_{1}, x_{2}, x_{3}\right)=\left(x_{1}, x_{2},-x_{3}\right)$ and $\boldsymbol{m}^{\sigma}=\boldsymbol{m} \circ \sigma$. The Euler-Lagrange stationary conditions for equilibrium states are $\boldsymbol{m} \wedge \boldsymbol{h}=\mathbf{0}$ in $\mathcal{O}$ and

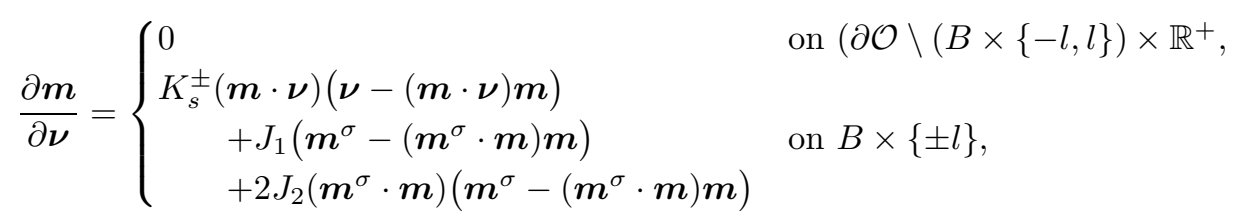

on the boundary. In the absence of surface energies, these boundary conditions simplify to

$$
\frac{\partial \boldsymbol{m}}{\partial \boldsymbol{\nu}}=0 \quad \text { on } \partial \mathcal{O} \times \mathbb{R}^{+} .
$$

Should there be more than one spacer, as it will be the case later in this paper, each spacer $i$ has its own set of constants $K_{s}^{+}, K_{s}^{-}, J_{1}, J_{2}$. The positive constants $K_{s}^{+}, K_{s}^{-}, J_{1}, J_{2}$ may also depend on the thickness of the interlayer $l$.

While the micromagnetic model fully characterizes the equilibrium states of the magnetization, a PDE is needed in order to have a dynamic model. This PDE was supplied by Landau and Lifshitz [12].

\subsection{The dynamic model: the Landau-Lifshitz system}

At the mesoscopic scale, the magnetization evolves in time according to the phenomenological Landau-Lifshitz equation [12],

$$
\frac{\partial \boldsymbol{m}}{\partial t}=-\boldsymbol{m} \wedge \boldsymbol{h}-\alpha \boldsymbol{m} \wedge(\boldsymbol{m} \wedge \boldsymbol{h}) \quad \text { in } \mathcal{O} \times \mathbb{R}^{+},
$$

where $\boldsymbol{h}$ is the magnetic effective field provided by the micromagnetic model.

The complete Landau-Lifshitz system is characterized by the non-convex constraint (2.1), the evolution equation (2.3), an initial condition

$$
\boldsymbol{m}(\cdot, 0)=\boldsymbol{m}_{0}
$$

and the boundary conditions (2.2) for every time $t$. The stationary boundary conditions are used to complete the dynamic model mainly because they allow to recover, at least formally, the energy inequality from the Landau-Lifshitz equation,

for all time $T>0$.

$$
\mathrm{E}(\boldsymbol{m}(\cdot, T))+\frac{\alpha}{1+\alpha^{2}} \int_{0}^{T}\left\|\frac{\partial \boldsymbol{m}}{\partial t}\right\|_{\mathrm{L}^{2}\left(\mathcal{O} ; \mathbb{R}^{3}\right)} \leq \mathrm{E}\left(\boldsymbol{m}_{0}\right)
$$

\section{Notations AND GeOMetries}

In this section, we describe the multilayer geometry and the notations that are used throughout the paper. Let $L>0$. Let $B$ be a convex open bounded subset of $\mathbb{R}^{2}$ with a smooth boundary. Let $Y=[0,1]$. Let $p$ be a positive nonzero integer. Given $p$ lengths $\theta_{0}, \ldots, \theta_{p-1}$, and $p$ positions $0=z_{0}<\ldots<z_{p-1}<1$, let $z_{i+k p}=z_{i}+k$, and $\theta_{i}=\theta_{i} \bmod p$ for $i$ in $\mathbb{Z}$. Let $z_{i}^{+}=z_{i}+\frac{\theta_{i}}{2}$, and $z_{i}^{-}=z_{i}-\frac{\theta_{i}}{2}$. We assume that $z_{i}^{+}<z_{i+1}^{-}$ 


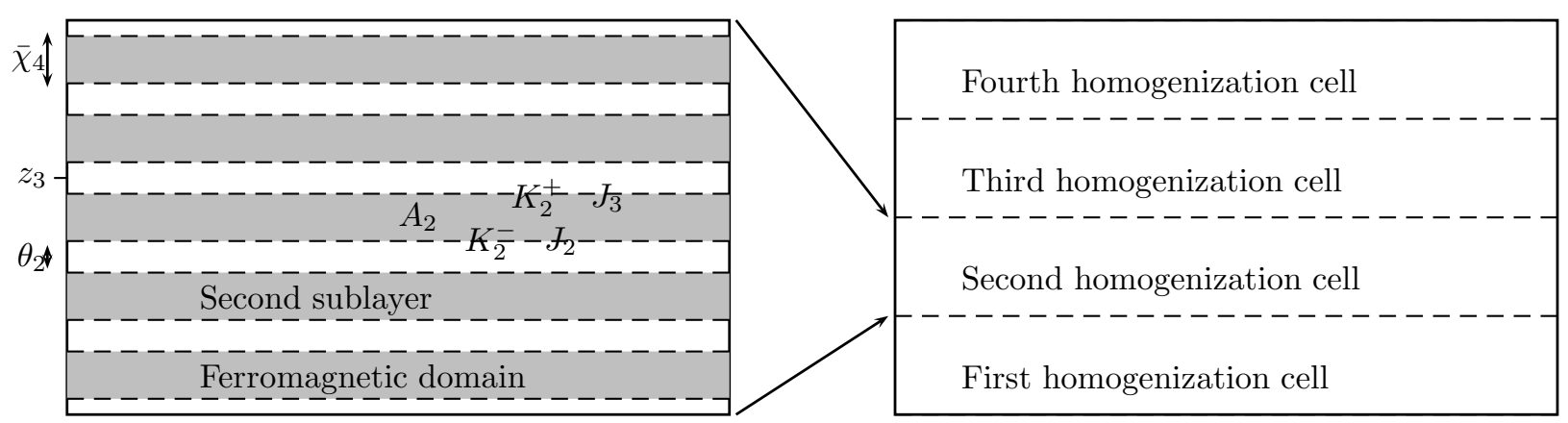

One homogenization cell

Multilayered domain $N=4$

Figure 1. Example of multilayer domain for $p=5$.

for $i$ in $\mathbb{Z}$. Let $\bar{\chi}_{i}=z_{i+1}^{-}-z_{i}^{+}$be the thickness of layer $i$, and $\bar{\chi}=\sum_{i=0}^{p-1} \bar{\chi}_{i}$ be the total thickness of the layers. Then, we define the intervals:

$$
\begin{aligned}
& I_{i}=\left(z_{i}^{+}, z_{i+1}^{-}\right), \\
& \widetilde{I}_{i}=\bigcup_{k \in \mathbb{Z}} I_{i+k p}, \\
& \widetilde{I}=\bigcup_{i \in \mathbb{Z}} I_{i}, \\
& I=\widetilde{I} \cap(0,1), \\
& J_{i}=\left(z_{i}^{-}, z_{i}^{+}\right), \\
& \widetilde{J}_{i}=\bigcup_{k \in \mathbb{Z}} J_{i+k p}, \\
& \widetilde{J}=\bigcup_{i \in \mathbb{Z}} J_{i}, \\
& J=\widetilde{J} \cap(0,1),
\end{aligned}
$$

and

$$
\begin{array}{llll}
\widetilde{I}_{i}^{N}=\frac{L}{N} \widetilde{I}_{i}, & \widetilde{I}^{N}=\bigcup_{i=0}^{p-1} \widetilde{I}_{i}^{N}, & I_{i}^{N}=\widetilde{I}_{i}^{N} \cap(0, L), & I^{N}=\widetilde{I}^{N} \cap(0, L), \\
\widetilde{J}_{i}^{N}=\frac{L}{N} \widetilde{J}_{i}, & \widetilde{J}^{N}=\bigcup_{i=0}^{p-1} \widetilde{J}_{i}^{N}, & J_{i}^{N}=\widetilde{J}_{i}^{N} \cap(0, L), & J^{N}=\widetilde{J}^{N} \cap(0, L),
\end{array}
$$

along with the domains:

$$
\begin{aligned}
\Omega=B \times(0, L), & \Omega^{N}=B \times I^{N}, & \Omega_{i}^{N}=B \times I_{i}^{N}, \\
Q_{T}=\Omega \times(0, T), & Q_{T}^{N}=\Omega^{N} \times(0, T), & Q_{T, i}^{N}=\Omega_{i}^{N} \times(0, T) .
\end{aligned}
$$

The domain $\Omega^{N}$ is the domain filled with ferromagnetic material when there are $N$ homogenization cells. Since super-exchange and surface anisotropy are present on the boundary, we define

$$
\begin{array}{rlrl}
\Gamma_{i, k}^{N} & =B \times\left\{\frac{L}{N} z_{i+k p}\right\}, & \Gamma_{i, k}^{N,+}=B \times\left\{\frac{L}{N} z_{i+k p}^{+}\right\}, & \Gamma_{i, k}^{N,-}=B \times\left\{\frac{L}{N} z_{i+k p}^{-}\right\}, \\
\Gamma_{i}^{N}=\bigcup_{k=\delta_{i}^{0}}^{N-1-\delta_{i}^{p-1}} \Gamma_{i, k}^{N}, & \Gamma_{i}^{N,+}=\bigcup_{k=\delta_{i}^{0}}^{N-1-\delta_{i}^{p-1}} \Gamma_{i, k}^{N,+}, & \Gamma_{i}^{N,-}=\bigcup_{k=\delta_{i}^{0}}^{N-1-\delta_{i}^{p-1}} \Gamma_{i, k}^{N,-},
\end{array}
$$

for all integers $i, k, 0 \leq i \leq p-1,0 \leq k \leq N-1$, and where $\delta$ is the Kronecker's symbol. The exterior and lateral boundaries are denoted by

$$
\Gamma_{l}^{N}=\partial B \times I^{N}, \quad \Gamma_{e}^{N}=\Gamma_{l}^{N} \cup \Gamma_{p-1, N-1}^{N,-} \cup \Gamma_{0,0}^{N,+},
$$


while the interior boundaries are

$$
\Gamma^{N,+}=\bigcup_{i=0}^{p-1} \Gamma_{i}^{N,+}, \quad \Gamma^{N,-}=\bigcup_{i=0}^{p-1} \Gamma_{i}^{N,-}, \quad \Gamma^{N}=\bigcup_{i=0}^{p-1} \Gamma_{i}^{N} .
$$

The vector $\boldsymbol{\nu}$ always represents the normal to the boundary $\Omega^{N}$ whose orientation points to the exterior of $\Omega^{N}$.

Now that the domains $\Omega^{N}$ are explicitly defined, we introduce various trace applications:

- Let $\gamma$ be the operator that maps any sufficiently regular function defined over $\Omega^{N}$ into its trace on $\Gamma^{N,+} \cup \Gamma^{N,-}$.

- Let $\sigma$ be a function that maps $\left(x_{1}, x_{2}, x_{3}\right)$ onto $\left(x_{1}, x_{2},-x_{3}\right)$, and $\tau_{\epsilon}$ maps $\left(x_{1}, x_{2}, x_{3}\right)$ onto $\left(x_{1}, x_{2}, x_{3}-\epsilon\right)$. Let $\gamma^{\prime}$ be the trace operators that maps any function $u$ into $\gamma u \circ \sigma \circ \tau_{2 \frac{L}{N} z_{i}}$ on $\Gamma_{i}^{N,+} \cup \Gamma_{i}^{N,-}$.

- Let $\gamma^{+}$be the trace operator that maps $u$ into $\gamma u \circ \tau_{-\frac{L}{2 N} \theta_{i}}$ on $\Gamma_{i}^{N}$ and $\gamma^{-}$be the trace operator that maps $u$ into $\gamma u \circ \tau_{\frac{L}{2 N} \theta_{i}}$ on $\Gamma_{i}^{N}$.

We finally introduce some characteristic functions.

- Let $\chi^{N}$ be the characteristic function of domain $\Omega^{N}$.

- Let $\chi$ be the characteristic function of interval $I$.

We introduce the spaces $\mathrm{H}_{\text {loc }}^{1}\left(\mathcal{O} \times \mathbb{R}^{+}\right)$which refer to the space of all measurable functions defined over $\mathcal{O} \times \mathbb{R}^{+}$ whose restriction to $\mathcal{O} \times(0, T)$ are $\mathrm{H}^{1}$ for all $T>0$. The space $\mathrm{L}_{\text {loc }}^{2}\left(\mathcal{O} \times \mathbb{R}^{+}\right)$contains all measurable functions defined over $\mathcal{O} \times \mathbb{R}^{+}$whose restriction to $\mathcal{O} \times(0, T)$ are $\mathrm{L}^{2}$ for all $T>0$. By extension, convergence to $u$ in $\mathrm{L}_{\text {loc }}^{2}\left(\mathcal{O} \times \mathbb{R}^{+}\right)$in any type of convergence (whether strong, weak, two-scale or any other possibility) should be understood as meaning that, for all $T>0$, the restriction to $\mathcal{O} \times(0, T)$ converges in this particular type to the restriction of $u$ to $\mathcal{O} \times(0, T)$.

\section{The Landau-Lifshitz EQUation in a multilayer}

We first describe explicitly the Landau-Lifshitz system in a periodic ferromagnetic multilayer. Then, we present the definition of weak solutions to the Landau-Lifshitz system.

\subsection{The strong formulation}

We consider a ferromagnet filling domain $\Omega^{N}$. To model quantitatively the behavior of this ferromagnet, we set the micromagnetic parameters for each layer. We assume that there are $p$ positive exchange constants $\left(A_{0}, \ldots, A_{p-1}\right), p$ symmetric positive anisotropy matrices, $\left(\mathbf{K}_{0}, \ldots, \mathbf{K}_{p-1}\right), 2 p$ surface anisotropy constants $\left(K_{s, 0}^{+}, \ldots, K_{s, p-1}^{+}\right)$and $\left(K_{s, 0}^{-}, \ldots, K_{s, p-1}^{-}\right), 2 p$ super-exchange constants $\left(J_{1,0}, \ldots, J_{1, p-1}\right)$ and $\left(J_{2,0}, \ldots, J_{2, p-1}\right)$ as well as two real parameters $\rho$ and $\varpi$ that control the variation of the magnitude of the super-exchange and surface anisotropy interactions, respectively, with respect to the interlayer distance.

We consider the following system of equations to be homogenized:

$$
\frac{\partial \boldsymbol{m}^{N}}{\partial t}=-\boldsymbol{m}^{N} \wedge\left(A_{i} \triangle \boldsymbol{m}^{N}-\mathbf{K}_{i} \boldsymbol{m}^{N}+\mathcal{H}_{d}\left(\boldsymbol{m}^{N}\right)\right)-\alpha \boldsymbol{m}^{N} \wedge\left(\boldsymbol{m}^{N} \wedge\left(A_{i} \triangle \boldsymbol{m}^{N}-\mathbf{K}_{i} \boldsymbol{m}^{N}+\mathcal{H}_{d}\left(\boldsymbol{m}^{N}\right)\right)\right),
$$

in $\Omega_{i}^{N} \times \mathbb{R}^{+}$for any integer $i, 0 \leq i \leq p-1$.

$$
\begin{aligned}
& \left|\boldsymbol{m}^{N}\right|=1 \quad \text { in } \Omega^{N} \times \mathbb{R}^{+}, \\
& \boldsymbol{m}^{N}(\cdot, 0)=\boldsymbol{m}_{0} \quad \text { in } \Omega^{N},
\end{aligned}
$$


and

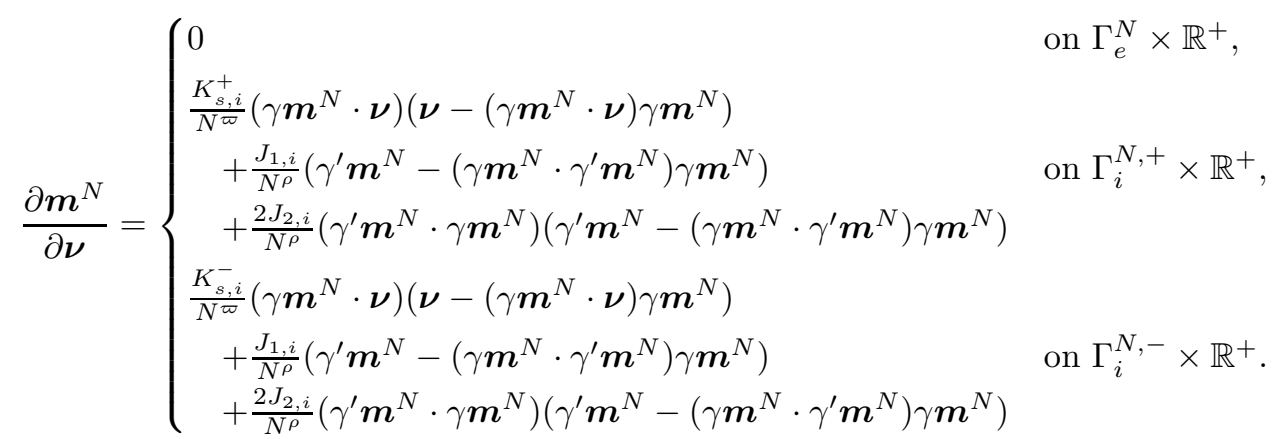

In system (4.1), only boundary conditions are scaled with respect to the number of homogenization cells $N$. This scaling is modeled through two parameters $\rho$ and $\varpi$. We will study two cases:

(1) In Section 6.1, $\varpi=0$ which corresponds to strong surface anisotropy interaction whose magnitude does not diminish when the number of layers $N p$ goes to infinity. There are two subcases: $\rho=0$ in Section 6.1 .1 and $\rho=1$ in Section 6.1.2, yielding slight different results.

(2) In Section $6.2, \varpi=1$ and $\rho=-1$ which corresponds to a fading surface anisotropy and an exploding super-exchange when the number of layers $N p$ goes to infinity.

We make the following remark on our model:

Remark 2. Ideally, the parameters $\alpha$ and $|\boldsymbol{m}|$ should also depend on the layer. To avoid adding unnecessary complications, we have chosen to let them remain constant.

\subsection{Weak solutions in a multilayer}

In this section, we present the definition of weak solutions to the Landau-Lifshitz system (4.1) in a multilayer.

First, note that, when constraint (4.1b) holds, then equation (4.1a) is, at least formally, equivalent to the Landau-Lifshitz-Gilbert equation

$$
\frac{\partial \boldsymbol{m}^{N}}{\partial t}-\alpha \boldsymbol{m}^{N} \wedge \frac{\partial \boldsymbol{m}^{N}}{\partial t}=-\left(1+\alpha^{2}\right) \boldsymbol{m}^{N} \wedge\left(A_{i} \triangle \boldsymbol{m}^{N}-\mathbf{K}_{i} \boldsymbol{m}^{N}+\mathcal{H}_{d}\left(\boldsymbol{m}^{N}\right)\right) .
$$

Definition 3. Let $\boldsymbol{m}_{0}^{N}$ be in $\mathrm{H}^{1}\left(\Omega^{N} ; \mathbb{R}^{3}\right),\left|\boldsymbol{m}_{0}^{N}\right|=1$ a.e. in $\Omega^{N}$, we say that $\boldsymbol{m}^{N}$ in $\mathrm{H}_{\mathrm{loc}}^{1}\left(\Omega^{N} \times \mathbb{R}^{+} ; \mathbb{R}^{3}\right) \cap$ $\mathrm{L}^{\infty}\left(0,+\infty ; \mathrm{H}^{1}\left(\Omega^{N} ; \mathbb{R}^{3}\right)\right)$ is a weak solution to the Landau-Lifshitz system if

(1) The non-convex constraint is satisfied, i.e,

$$
\left|\boldsymbol{m}^{N}\right|=1 \quad \text { a.e. in } \Omega^{N} \times \mathbb{R}^{+} .
$$

(2) For all time $T>0$, for all $\phi$ in $\mathcal{C}^{\infty}\left(\overline{\Omega^{N}} \times[0, T] ; \mathbb{R}^{3}\right)$,

$$
\begin{aligned}
& \iint_{\Omega^{N} \times(0, T)} \frac{\partial \boldsymbol{m}^{N}}{\partial t} \cdot \boldsymbol{\phi} \mathrm{d} \boldsymbol{x} \mathrm{d} t-\alpha \iint_{\Omega^{N} \times(0, T)}\left(\boldsymbol{m}^{N} \wedge \frac{\partial \boldsymbol{m}^{N}}{\partial t}\right) \cdot \boldsymbol{\phi} \mathrm{d} \boldsymbol{x} \mathrm{d} t \\
&=\left(1+\alpha^{2}\right) \sum_{i=0}^{p-1} A_{i} \iint_{\Omega_{i}^{N} \times(0, T)} \sum_{j=1}^{3}\left(\boldsymbol{m}^{N} \wedge \frac{\partial \boldsymbol{m}^{N}}{\partial x_{j}}\right) \cdot \frac{\partial \boldsymbol{\phi}}{\partial x_{j}} \mathrm{~d} \boldsymbol{x} \mathrm{d} t \\
&-\left(1+\alpha^{2}\right) \iint_{\Omega^{N} \times(0, T)}\left(\boldsymbol{m}^{N} \wedge \mathcal{H}_{d}\left(\boldsymbol{m}^{N}\right)\right) \cdot \boldsymbol{\phi} \mathrm{d} \boldsymbol{x} \mathrm{d} t \\
&+\left(1+\alpha^{2}\right) \sum_{i=0}^{p-1} \iint_{\Omega_{i}^{N} \times(0, T)}\left(\boldsymbol{m}^{N} \wedge \mathbf{K}_{i} \boldsymbol{m}^{N}\right) \cdot \boldsymbol{\phi} \mathrm{d} \boldsymbol{x} \mathrm{d} t
\end{aligned}
$$




$$
\begin{aligned}
& -\left(1+\alpha^{2}\right) \sum_{i=0}^{p-1} \frac{K_{s, i}^{+}}{N^{\varpi}} \iint_{\Gamma_{i}^{N,+} \times(0, T)}\left(\boldsymbol{\nu} \cdot \gamma \boldsymbol{m}^{N}\right)\left(\gamma \boldsymbol{m}^{N} \wedge \boldsymbol{\nu}\right) \cdot \boldsymbol{\phi} \mathrm{d} \boldsymbol{x} \mathrm{d} t \\
& -\left(1+\alpha^{2}\right) \sum_{i=0}^{p-1} \frac{K_{s, i}^{-}}{N^{\varpi}} \iint_{\Gamma_{i}^{N,-} \times(0, T)}\left(\boldsymbol{\nu} \cdot \gamma \boldsymbol{m}^{N}\right)\left(\gamma \boldsymbol{m}^{N} \wedge \boldsymbol{\nu}\right) \cdot \boldsymbol{\phi} \mathrm{d} \boldsymbol{x} \mathrm{d} t \\
& -\left(1+\alpha^{2}\right) \sum_{i=0}^{p-1}\left(\frac{J_{1, i}}{N^{\rho}} \iint_{\Gamma_{i}^{N} \times(0, T)}\left(\gamma^{+} \boldsymbol{m}^{N} \wedge \gamma^{-} \boldsymbol{m}^{N}\right) \cdot\left(\gamma^{+} \boldsymbol{\phi}-\gamma^{-} \boldsymbol{\phi}\right) \mathrm{d} \boldsymbol{x} \mathrm{d} t\right. \\
& \left.+2 \frac{J_{2, i}}{N^{\rho}} \iint_{\Gamma_{i}^{N} \times(0, T)}\left(\gamma^{+} \boldsymbol{m}^{N} \cdot \gamma^{-} \boldsymbol{m}^{N}\right)\left(\gamma^{+} \boldsymbol{m}^{N} \wedge \gamma^{-} \boldsymbol{m}^{N}\right) \cdot\left(\gamma^{+} \boldsymbol{\phi}-\gamma^{-} \phi\right)\right) \mathrm{d} \boldsymbol{x} \mathrm{d} t .
\end{aligned}
$$

(3) On $\Omega^{N} \times\{0\}$, in the sense of traces,

$$
\boldsymbol{m}^{N}(\cdot, 0)=\boldsymbol{m}_{0}^{N}
$$

(4) For all time $T>0$, the following energy inequality is satisfied:

$$
\mathrm{E}^{N}\left(\boldsymbol{m}^{N}(\cdot, T)\right)+\frac{\alpha}{1+\alpha^{2}} \int_{0}^{T}\left\|\frac{\partial \boldsymbol{m}^{N}}{\partial t}\right\|_{L^{2}\left(\Omega^{N}\right)}^{2} \mathrm{~d} t \leq \mathrm{E}^{N}\left(\boldsymbol{m}_{0}^{N}\right)
$$

where

$$
\begin{aligned}
\mathrm{E}^{N}(\boldsymbol{u})= & \sum_{i=0}^{p-1} \frac{A_{i}}{2}\|\nabla \boldsymbol{u}\|_{\mathrm{L}^{2}\left(\Omega_{i}^{N}\right)}^{2}+\frac{1}{2} \int_{\mathbb{R}^{3}}\left|\mathcal{H}_{d}(\boldsymbol{u})\right|^{2} \mathrm{~d} \boldsymbol{x} \\
& +\frac{1}{2} \sum_{i=0}^{p-1} \int_{\Omega_{i}^{N}} \boldsymbol{u} \cdot\left(\mathbf{K}_{i} \boldsymbol{u}\right) \mathrm{d} \boldsymbol{x}+\sum_{i=0}^{p-1} \frac{K_{s, i}^{+}}{2 N^{\varpi}} \int_{\Gamma_{i}^{N,+}}|\gamma \boldsymbol{u} \wedge \boldsymbol{\nu}|^{2} \mathrm{~d} \sigma(\boldsymbol{x}) \\
& +\sum_{i=0}^{p-1} \frac{K_{s, i}^{-}}{2 N^{\varpi}} \int_{\Gamma_{i}^{N,-}}|\gamma \boldsymbol{u} \wedge \boldsymbol{\nu}|^{2} \mathrm{~d} \sigma(\boldsymbol{x})+\sum_{i=0}^{p-1} \frac{J_{1, i}}{2 N^{\rho}} \int_{\Gamma_{i}^{N}}\left|\gamma^{+} \boldsymbol{u}-\gamma^{-} \boldsymbol{u}\right|^{2} \mathrm{~d} \sigma(\boldsymbol{x}) \\
& +\sum_{i=0}^{p-1} \frac{J_{2, i}}{N^{\rho}} \int_{\Gamma_{i}^{N}}\left|\gamma^{+} \boldsymbol{u} \wedge \gamma^{-} \boldsymbol{u}\right|^{2} \mathrm{~d} \sigma(\boldsymbol{x}) .
\end{aligned}
$$

For any admissible initial condition, there exists at least one global weak solution, but no uniqueness result ${ }^{3}$ :

Theorem 4. Let $\boldsymbol{m}_{0}$ be in $\mathrm{H}^{1}\left(\Omega^{N} ; \mathbb{R}^{3}\right)\left|\boldsymbol{m}_{0}\right|=1$ a.e. in $\Omega^{N}$. There exists at least one weak solution to the Landau-Lifshitz system in the sense of Definition 3.

Proof. See Theorem 3.2 in [15] for two layers. The proof generalizes itself without difficulty to a finite number of layers.

\section{The TWO-SCALE CONVERGENCE}

This section briefly recalls the important notion of two-scale convergence, described in Allaire [2], in Allaire, Damlamian and Hornung [3], and in Neuss-Radu [13,14]. Throughout this section, $\mathcal{O}$ is an open set of $\mathbb{R}^{3}$, and $Y=[0,1]$. Let $\mathcal{C}_{\#}^{\infty}(Y)$ be the space of 1-periodic indefinitely differentiable functions in $\mathbb{R}$. Let $\mathrm{H}_{\#}^{1}(Y)$ be the

\footnotetext{
${ }^{3}$ All known uniqueness results for weak solution to the Landau-Lifshitz system in 3 dimensions are negative, see Alouges and Soyeur [4].
} 
closure of $\mathcal{C}_{\#}^{\infty}(Y)$ in $\mathrm{H}^{1}(Y)$. Let $\mathcal{C}^{\infty}(\overline{\mathcal{O}}) \otimes \mathcal{C}_{\#}^{\infty}(Y)$ be the space of indefinitely differentiable functions on $\overline{\mathcal{O}} \times \mathbb{R}$, which are 1-periodic on the last variable. Let $\mathrm{H}_{\#}^{1}(\mathcal{O} \times Y)$ be the closure of $\mathcal{C}^{\infty}(\mathcal{O}) \otimes \mathcal{C}_{\#}^{\infty}(Y)$ in $\mathrm{H}^{1}(\mathcal{O} \times Y)$.

Definition 5. A sequence of functions $u_{\epsilon}$ in $\mathrm{L}^{2}(\mathcal{O})$ is said to two-scale converge to a limit $u_{0}(\boldsymbol{x}, y)$ in $\mathrm{L}^{2}(\mathcal{O} \times Y)$ if

$$
\lim _{\epsilon \rightarrow 0} \int_{\mathcal{O}} u_{\epsilon}(\boldsymbol{x}) \psi\left(\boldsymbol{x}, \frac{x_{3}}{\epsilon}\right) \mathrm{d} \boldsymbol{x}=\int_{\mathcal{O}} \int_{Y} u_{0}(\boldsymbol{x}, y) \psi(\boldsymbol{x}, y) \mathrm{d} y \mathrm{~d} \boldsymbol{x},
$$

for all $\psi$ in $\mathcal{C}^{\infty}(\overline{\mathcal{O}}) \otimes \mathcal{C}_{\#}^{\infty}(Y)$.

We reproduce the compactness Theorem 1.2 in [2]:

Theorem 6. Let $u_{\epsilon}$ be a bounded sequence of elements bounded in $\mathrm{L}^{2}(\mathcal{O})$, then there exists a subsequence $\left(\epsilon_{k}\right)_{k \in \mathbb{N}}$, and $u_{0}$ in $\mathrm{L}^{2}(\mathcal{O} \times Y)$ such that $u_{\epsilon_{k}}$ two-scale converges to $u_{0}$.

We recall a simple criterion that justifies the convergence of products.

Theorem 7. Let $u_{\epsilon}$ and $v_{\epsilon}$ be bounded sequences in $\mathrm{L}^{2}(\mathcal{O})$ that two-scale converge to $u_{0}$ and $v_{0}$, respectively in $\mathrm{L}^{2}(\mathcal{O} \times Y)$. If

$$
\left\|u_{0}\right\|_{\mathrm{L}^{2}(\mathcal{O} \times Y)}=\liminf _{\epsilon \rightarrow 0}\left\|u_{\epsilon}\right\|_{\mathrm{L}^{2}(\mathcal{O})}
$$

then

$$
\lim _{\epsilon \rightarrow 0} \int_{\mathcal{O}} u_{\epsilon}(\boldsymbol{x}) v_{\epsilon}(\boldsymbol{x}) \psi\left(\boldsymbol{x}, \frac{x_{3}}{\epsilon}\right) \mathrm{d} \boldsymbol{x}=\int_{\mathcal{O}} \int_{Y} u_{0}(\boldsymbol{x}, y) v_{0}(\boldsymbol{x}, y) \psi(\boldsymbol{x}, y) \mathrm{d} y \mathrm{~d} \boldsymbol{x},
$$

for all $\psi$ in $\mathcal{C}^{\infty}(\overline{\mathcal{O}}) \otimes \mathcal{C}_{\#}^{\infty}(Y)$.

Proof. See [2] Theorem 1.8.

We describe the concept of two-scale convergence with periodic surfaces, see Allaire, Damlamian and Hornung [3], and M. Neuss-Radu [13,14]. Let $Y=[0,1], T$ be an open set of $Y$, and $\Gamma$ its boundary. We define $Y^{*}=Y \backslash T$. Let

$$
\Gamma^{N}=\left\{\boldsymbol{x} \in \mathcal{O}, N x_{3} \in \Gamma+\mathbb{Z}\right\}, \quad \mathcal{O}^{N}=\left\{\boldsymbol{x} \in \mathcal{O}, N x_{3} \in Y^{*}+\mathbb{Z}\right\} .
$$

Theorem 8. Let $u^{N}$ be a sequence of functions of $\mathrm{L}^{2}\left(\Gamma^{N}\right)$ such that

$$
\frac{1}{N} \int_{\Gamma^{N}}\left|u^{N}(\boldsymbol{x})\right|^{2} \mathrm{~d} \sigma(\boldsymbol{x}) \leq C,
$$

where $C$ is a constant. There exists a subsequence $N_{k}$, and $u^{\infty}$ in $\mathrm{L}^{2}(\mathcal{O} \times \Gamma)$, such that

$$
\lim _{k \rightarrow \infty} \frac{1}{N_{k}} \int_{\Gamma^{N_{k}}} u^{N_{k}}(\boldsymbol{x}) \phi\left(\boldsymbol{x}, N x_{3}\right) \mathrm{d} \sigma(\boldsymbol{x})=\int_{\mathcal{O}} \int_{\Gamma} u^{\infty}(\boldsymbol{x}, y) \phi(\boldsymbol{x}, y) \mathrm{d} \sigma(y) \mathrm{d} \boldsymbol{x},
$$

for all $\phi$ in $\mathcal{C}(\overline{\mathcal{O}}) \otimes \mathcal{C}_{\#}(Y)$.

Proof. See Theorem 1.1 in [3].

The relation (5.3) defines the two-scale convergence with periodic surfaces. Under, some assumptions, there exists a link between the two forms of two-scale convergence.

Proposition 9. Let $u^{N}$ be a sequence of functions in $\mathrm{H}^{1}(\mathcal{O})$ such that

$$
\left\|u^{N}\right\|_{\mathrm{L}^{2}(\mathcal{O})}+\frac{1}{N}\left\|\nabla u^{N}\right\|_{\mathrm{L}^{2}(\mathcal{O})} \leq C
$$


where $C>0$ is a constant. Then, the trace of $u^{N}$ on $\Gamma^{N}$ satisfies

$$
\frac{1}{N} \int_{\Gamma^{N}}\left|u^{N}(\boldsymbol{x})\right|^{2} \mathrm{~d} \sigma(\boldsymbol{x}) \leq C,
$$

and there exists $u^{\infty}$ in $\mathrm{L}^{2}\left(\mathcal{O} ; \mathrm{H}_{\#}^{1}(Y)\right)$ and a subsequence $N_{k}$, such that

$$
\begin{gathered}
\lim _{k \rightarrow \infty} \frac{1}{N_{k}} \int_{\Gamma^{N_{k}}} u^{N_{k}}(\boldsymbol{x}) \phi\left(\boldsymbol{x}, N_{k} x_{3}\right) \mathrm{d} \sigma(\boldsymbol{x}) \\
=\int_{\mathcal{O}} \int_{\Gamma} u^{\infty}(\boldsymbol{x}, y) \phi(\boldsymbol{x}, y) \mathrm{d} \boldsymbol{x} \mathrm{d} \sigma(y), \\
\lim _{k \rightarrow \infty} \int_{\mathcal{O}} u^{N_{k}}(\boldsymbol{x}) \psi\left(\boldsymbol{x}, N_{k} x_{3}\right) \mathrm{d} \boldsymbol{x}=\int_{\mathcal{O}} \int_{Y} u^{\infty}(\boldsymbol{x}, y) \psi(\boldsymbol{x}, y) \mathrm{d} \boldsymbol{x} \mathrm{d} y, \\
\lim _{k \rightarrow \infty} \frac{1}{N_{k}} \int_{\mathcal{O}} \frac{\partial u^{N_{k}}}{\partial x_{3}}(\boldsymbol{x}) \psi\left(\boldsymbol{x}, N_{k} x_{3}\right) \mathrm{d} \boldsymbol{x}=\int_{\mathcal{O}} \int_{Y} \frac{\partial u^{\infty}}{\partial y}(\boldsymbol{x}, y) \psi(\boldsymbol{x}, y) \mathrm{d} \boldsymbol{x} \mathrm{d} y,
\end{gathered}
$$

for all $\psi$ in $\mathcal{C}(\overline{\mathcal{O}}) \otimes \mathcal{C}_{\#}(Y)$.

Proof. See Proposition 2.6 in [3].

The following theorem has analogous result to Theorem 7 for the two-scale convergence with periodic surfaces.

Theorem 10. Let $u^{N}$ and $v^{N}$ be two sequences bounded in $\mathrm{L}^{2}(\mathcal{O})$, two-scale converging to respectively $u^{\infty}$ and $v^{\infty}$ in the sense of (5.3). If

$$
\lim _{N \rightarrow \infty} \frac{1}{N} \int_{\Gamma^{N}}\left|u^{N}(\boldsymbol{x})\right|^{2} \mathrm{~d} \sigma(\boldsymbol{x})=\int_{\mathcal{O}} \int_{\Gamma}\left|u^{\infty}(\boldsymbol{x}, y)\right|^{2} \mathrm{~d} \sigma(y) \mathrm{d} \boldsymbol{x},
$$

then, for all $\phi$ in $\mathcal{C}^{\infty}(\overline{\mathcal{O}}) \otimes \mathcal{C}_{\#}^{\infty}(Y)$,

$$
\lim _{N \rightarrow \infty} \frac{1}{N} \int_{\Gamma^{N}} u^{N}(\boldsymbol{x}) v^{N}(\boldsymbol{x}) \phi\left(\boldsymbol{x}, N x_{3}\right) \mathrm{d} \sigma(\boldsymbol{x})=\int_{\mathcal{O}} \int_{\Gamma} u^{\infty}(\boldsymbol{x}, y) v^{\infty}(\boldsymbol{x}, y) \phi(\boldsymbol{x}, y) \mathrm{d} \sigma(y) \mathrm{d} \boldsymbol{x} .
$$

Proof. It is left to the reader to verify that the proof of Theorem 1.8 in [2] can easily be adapted.

Using the different concepts of two-scale convergence recalled in this section, we can establish the homogenized equations for ferromagnetic multilayers.

\section{The homogenized Landau-Lifshitz Equation}

In this section, we present the equations satisfied by two-scale limits of subsequences of weak solutions $\boldsymbol{m}^{N}$ to the Landau-Lifshitz system for different values of the parameters $\varpi$ and $\rho$. We always assume that the sequence of initial conditions $\boldsymbol{m}_{0}^{N}$ satisfies

$$
\sup _{N \in \mathbb{N}} \mathrm{E}^{N}\left(\boldsymbol{m}_{0}^{N}\right) \leq+\infty
$$

where $\mathrm{E}^{N}$ is the functional given by (4.3). 


\subsection{Strong surface anisotropy}

In this section, we assume that the magnitude of surface anisotropy does not depend on the interlayer thickness, i.e. $\varpi=0$. In that case, surface anisotropy dominates all other phenomena and forces the limit to be stationary. We study two subcases: $\rho=0$ and $\rho=1$, where $\rho$ is the exponent modeling the variation of the super-exchange constant with respect to the number of homogenization cells $N$, i.e. the magnitude of the super-exchange for the quadratic term and biquadratic term are $\frac{J_{1, i}}{N^{\rho}}$ and $\frac{J_{2, i}}{N^{\rho}}$, respectively. Throughout this section, let $\boldsymbol{e}_{3}$ be the vector $(0,0,1)$.

We will need a particular estimate of the $\mathrm{L}^{2}\left(\Omega^{N}\right)$ norm of a function:

Lemma 11. There exists $C>0$ independent of $N$ such that for all $u$ in $\mathrm{H}^{1}\left(\Omega^{N}\right)$ :

$$
\|u\|_{\mathrm{L}^{2}\left(\Omega^{N}\right)}^{2} \leq C\left(\frac{\|u\|_{\mathrm{L}^{2}\left(\Gamma^{N},+\cup \Gamma^{N,-}\right)}^{2}}{N}+\frac{\left\|\frac{\partial u}{\partial x_{3}}\right\|_{\mathrm{L}^{2}\left(\Omega^{N}\right)}^{2}}{N^{2}}\right) .
$$

Proof. We compute

$$
\begin{aligned}
\int_{\Omega^{N}}|u(\boldsymbol{x})|^{2} \mathrm{~d} \boldsymbol{x}=\sum_{i=0}^{p-1} \sum_{k=0}^{N-1} \int_{B} \int_{\frac{L}{N}\left(z_{i}^{+}+k\right)}^{\frac{L}{N}\left(z_{i+1}^{-}+k\right)}|u((\hat{\boldsymbol{x}}, z), T)|^{2} \mathrm{~d} z \mathrm{~d} \hat{\boldsymbol{x}} \\
\quad \leq \sum_{i=0}^{p-1} \sum_{k=0}^{N-1} \int_{\Gamma_{i, k}^{N,+}} \int_{0}^{\frac{L}{N} \bar{\chi}_{i}}\left|u\left(\boldsymbol{x}+z \boldsymbol{e}_{3}, T\right)\right|^{2} \mathrm{~d} z \mathrm{~d} \sigma(\boldsymbol{x}) \\
\quad \leq \sum_{i=0}^{p-1} \sum_{k=0}^{N-1} \int_{\Gamma_{i, k}^{N,+}} \int_{0}^{\frac{L}{N} \bar{\chi}_{i}}\left|\gamma u(\boldsymbol{x}, T)+\int_{0}^{z} \frac{\partial u}{\partial x_{3}}\left(\boldsymbol{x}+s \boldsymbol{e}_{3}, T\right) \mathrm{d} s\right|^{2} \mathrm{~d} z \mathrm{~d} \sigma(\boldsymbol{x}) .
\end{aligned}
$$

6.1.1. The case of $\rho=0$

In this section, we present one of our three theorems concerning the homogenization of the Landau-Lifshitz equation in multilayers. In this theorem, the initial conditions will be required to satisfy inequality (6.1). This represents a huge constraint on the possible initial magnetization. Before stating and proving this theorem, we feel important to provide the reader with one possible example of such initial conditions: we may choose $\boldsymbol{m}_{0}^{N}$ constant in each subcell and, to nullify surface anisotropy, equal to either $\boldsymbol{e}_{3}$ or $-\boldsymbol{e}_{3}$ everywhere. In this subcase, the super-exchange energy then forces the number of sign changes to remain bounded independently of $n$ in this subcase.

Theorem 12. Let $\varpi=0$ and $\rho=0$. Let $\boldsymbol{m}_{0}^{N}$ be a sequence of initial conditions in $\mathrm{H}^{1}\left(\Omega^{N} ; \mathbb{R}^{3}\right)$, $\left|\boldsymbol{m}_{0}^{N}\right|=1$ a.e. in $\Omega^{N}$, satisfying the energy constraint (6.1). Let $\boldsymbol{m}^{N}$ be a sequence of weak solutions to the Landau-Lifshitz system in the sense of Definition 3 . Then, there exists a sequence of extensions to $\boldsymbol{m}^{N}$ in $\mathrm{H}_{\mathrm{loc}}^{1}\left(\Omega \times \mathbb{R}^{/} ; \mathbb{R}^{3}\right)$ denoted $\widetilde{\boldsymbol{m}}^{N}$ and a subsequence of $\left(\widetilde{\boldsymbol{m}}^{N}\right)_{N}$ that two-scale converges to $\widetilde{\boldsymbol{m}}^{\infty}$ in $\mathrm{L}_{\text {loc }}^{2}\left(\Omega \times \mathbb{R}^{+} ; \mathbb{R}^{3}\right)$. The limit $\widetilde{\boldsymbol{m}}^{\infty}$ is independent of time and is parallel to $\boldsymbol{e}_{3}$ a.e. in $\Omega \times \mathbb{R}^{+}$.

Moreover, let $\overline{\boldsymbol{m}}_{0}^{N}$ be the extension by 0 of $\boldsymbol{m}_{0}^{N}$ outside $\Omega^{N}$. If there exists $\boldsymbol{m}_{0}^{\infty}$ in $\mathrm{L}^{2}(\Omega)$ such that $\overline{\boldsymbol{m}}_{0}^{N}$ converges weakly to $\bar{\chi} \boldsymbol{m}_{0}^{\infty}$ in $\mathrm{L}^{2}(\Omega)$, then the whole sequence converges and $\widetilde{\boldsymbol{m}}^{\infty}(\cdot, t)=\boldsymbol{m}_{0}^{\infty}$ for all time $t \geq 0$.

Proof. According to inequality (4.2d) and constraint (6.1), if $\rho=0$, then

$$
\begin{aligned}
\left\|\boldsymbol{m}^{N}\right\|_{\mathrm{L}^{\infty}\left(\mathbb{R}^{+} ; \mathrm{H}^{1}\left(\Omega^{N} ; \mathbb{R}^{3}\right)\right)}^{2} \leq C, & \left\|\frac{\partial \boldsymbol{m}^{N}}{\partial t}\right\|_{\mathrm{L}^{2}\left(\Omega^{N} \times \mathbb{R}^{+} ; \mathbb{R}^{3}\right)}^{2} \leq C, \\
\left\|\gamma^{+} \boldsymbol{m}^{N}-\gamma^{-} \boldsymbol{m}^{N}\right\|_{\mathrm{L}^{\infty}\left(\mathbb{R}^{+} ; \mathrm{L}^{2}\left(\Gamma^{N} ; \mathbb{R}^{3}\right)\right)}^{2} \leq C . &
\end{aligned}
$$


According to Proposition 23, there exists an extension of $\boldsymbol{m}^{N}$ over $\Omega \times \mathbb{R}^{+}$, denoted $\widetilde{\boldsymbol{m}}^{N}$ that satisfies

$$
\begin{aligned}
\left\|\widetilde{\boldsymbol{m}}^{N}\right\|_{\mathrm{L}^{\infty}\left(\Omega \times \mathbb{R}^{+} ; \mathbb{R}^{3}\right)} & \leq 1, & \left\|\frac{\partial \widetilde{\boldsymbol{m}}^{N}}{\partial t}\right\|_{\mathrm{L}^{2}\left(\Omega \times \mathbb{R}^{+} ; \mathbb{R}^{3}\right)} \leq C, \\
\left\|\frac{\partial \widetilde{\boldsymbol{m}}^{N}}{\partial x_{1}}\right\|_{L^{\infty}\left(\mathbb{R}^{+} ; \mathrm{L}^{2}\left(\Omega ; \mathbb{R}^{3}\right)\right)} & \leq C, & \left\|\frac{\partial \widetilde{\boldsymbol{m}}^{N}}{\partial x_{2}}\right\|_{L^{\infty}\left(\mathbb{R}^{+} ; \mathrm{L}^{2}\left(\Omega ; \mathbb{R}^{3}\right)\right)} \leq C, \\
\left\|\frac{\partial \widetilde{\boldsymbol{m}}^{N}}{\partial x_{3}}\right\|_{L^{\infty}\left(\mathbb{R}^{+} ; \mathrm{L}^{2}\left(\Omega ; \mathbb{R}^{3}\right)\right)} & \leq C \sqrt{N} . &
\end{aligned}
$$

According to Theorem 6 , there exists $\widetilde{\boldsymbol{m}}^{\infty}$ in $\mathrm{L}_{\mathrm{loc}}^{2}\left(\Omega \times \mathbb{R}^{+} \times(0,1)\right)$, such that for a subsequence $\widetilde{\boldsymbol{m}}^{N_{k}}$ :

$$
\lim _{k \rightarrow \infty} \iint_{\Omega \times(0, T)} \widetilde{\boldsymbol{m}}^{N_{k}}(\boldsymbol{x}, t) \phi\left(\boldsymbol{x}, t, \frac{N_{k}}{L} x_{3}\right) \mathrm{d} \boldsymbol{x} \mathrm{d} t=\iint_{\Omega \times(0, T)} \widetilde{\boldsymbol{m}}^{\infty}(\boldsymbol{x}, t) \int_{Y} \phi(\boldsymbol{x}, t, y) \mathrm{d} y \mathrm{~d} \boldsymbol{x} \mathrm{d} t
$$

for all time $T>0$ and for all $\phi$ in $\mathcal{C}^{\infty}(\bar{\Omega} \times[0, T]) \otimes \mathcal{C}^{\infty}(Y)$.

According to inequality (6.2c) and Proposition 1.14 in [2], $\widetilde{\boldsymbol{m}}^{\infty}$ does not depend on the fast variable $y$.

Since the two quantities $\left\|\boldsymbol{m}^{N} \wedge \boldsymbol{e}_{3}\right\|_{\mathrm{L}^{2}\left(\Gamma^{N,+} \cup \Gamma^{N,-}\right)}^{2}$ and $\left\|\frac{\partial \boldsymbol{m}^{N}}{\partial x_{3}}\right\|_{\mathrm{L}^{2}\left(\Omega^{N}\right)}^{2}$ are bounded by $\mathrm{E}^{N}\left(\boldsymbol{m}_{0}^{N}\right)$, Lemma 11 and inequality (4.2d) imply that

$$
\left\|\boldsymbol{m}^{N} \wedge \boldsymbol{e}_{3}\right\|_{\mathrm{L}^{\infty}\left(\mathbb{R}^{+} ; \mathrm{L}^{2}\left(\Omega^{N} ; \mathbb{R}^{3}\right)\right)}^{2} \leq \frac{C \mathrm{E}^{N}\left(\boldsymbol{m}_{0}^{N}\right)}{N}
$$

for some constant $C>0$. Since the sequence of initial conditions satisfy the energy boundedness (6.1), there exists $C^{\prime}>0$, independent of $N$, such that

$$
\left\|\boldsymbol{m}^{N} \wedge \boldsymbol{e}_{3}\right\|_{\mathrm{L}^{\infty}\left(\mathbb{R}^{+} ; \mathrm{L}^{2}\left(\Omega^{N} ; \mathbb{R}^{3}\right)\right)} \leq \frac{C^{\prime}}{\sqrt{N}}
$$

Therefore, $\widetilde{\boldsymbol{m}}^{\infty} \wedge \boldsymbol{e}_{3}=0$ a.e. in $\Omega \times \mathbb{R}+$.

Let $T>0$ and $\boldsymbol{\phi}=\phi \boldsymbol{e}_{3}$, where $\phi$ belongs to $\mathcal{C}^{\infty}(\bar{\Omega} \times[0, T])$, in $(4.2 \mathrm{~b})$ :

$$
\begin{aligned}
& \iint_{Q_{T}^{N}} \frac{\partial\left(\widetilde{\boldsymbol{m}}^{N} \cdot \boldsymbol{e}_{3}\right)}{\partial t} \phi \mathrm{d} \boldsymbol{x} \mathrm{d} t+ \alpha \underbrace{\iint_{Q_{T}^{N}}\left(\widetilde{\boldsymbol{m}}^{N} \wedge \boldsymbol{e}_{3}\right) \cdot \frac{\partial \widetilde{\boldsymbol{m}}^{N}}{\partial t} \phi \mathrm{d} \boldsymbol{x} \mathrm{d} t}_{I} \\
&=-\left(1+\alpha^{2}\right) \underbrace{\sum_{i=0}^{p-1} \sum_{j=1}^{3} A_{i} \iint_{Q_{T, i}^{N}}\left(\widetilde{\boldsymbol{m}}^{N} \wedge \boldsymbol{e}_{3}\right) \cdot \frac{\partial \widetilde{\boldsymbol{m}}^{N}}{\partial x_{j}} \frac{\partial \phi}{\partial x_{j}} \mathrm{~d} \boldsymbol{x} \mathrm{d} t}_{I I} \\
&+\left(1+\alpha^{2}\right) \underbrace{\iint_{Q_{T}^{N}}\left(\widetilde{\boldsymbol{m}}^{N} \wedge \boldsymbol{e}_{3}\right) \cdot \mathcal{H}_{d}\left(\widetilde{\boldsymbol{m}}^{N}\right) \phi \mathrm{d} \boldsymbol{x} \mathrm{d} t}_{I I I} \\
&-\left(1+\alpha^{2}\right) \underbrace{\sum_{i=0}^{p-1} \iint_{Q_{T, i}^{N}}\left(\widetilde{\boldsymbol{m}}^{N} \wedge \boldsymbol{e}_{3}\right) \cdot \mathbf{K}_{i} \widetilde{\boldsymbol{m}}^{N} \phi \mathrm{d} \boldsymbol{x} \mathrm{d} t}_{I V}
\end{aligned}
$$




$$
\begin{gathered}
+\left(1+\alpha^{2}\right) \underbrace{\sum_{i=0}^{p-1} J_{1, i} \iint_{\Gamma_{i}^{N} \times(0, T)}\left(\gamma^{+} \widetilde{\boldsymbol{m}}^{N} \wedge \boldsymbol{e}_{3}\right) \cdot \gamma^{-} \widetilde{\boldsymbol{m}}^{N}\left(\gamma^{+} \phi-\gamma^{-} \phi\right) \mathrm{d} \boldsymbol{x} \mathrm{d} t}_{V} \\
+2\left(1+\alpha^{2}\right) \underbrace{\sum_{i=0}^{p-1} J_{2, i} \iint_{\Gamma_{i}^{N} \times(0, T)}\left(\gamma^{+} \widetilde{\boldsymbol{m}}^{N} \cdot \gamma^{-} \widetilde{\boldsymbol{m}}^{N}\right)\left(\gamma^{+} \widetilde{\boldsymbol{m}}^{N} \wedge \boldsymbol{e}_{3}\right) \cdot \gamma^{-} \widetilde{\boldsymbol{m}}^{N}\left(\gamma^{+} \phi-\gamma^{-} \phi\right) \mathrm{d} \boldsymbol{x} \mathrm{d} t}_{V I},
\end{gathered}
$$

for all $\phi$ belonging to $\mathcal{C}^{\infty}(\bar{\Omega} \times[0, T])$. The surface anisotropy terms have vanished because $(\boldsymbol{a} \wedge \boldsymbol{\nu}) \cdot \boldsymbol{e}_{3}=0$. According to inequality (6.3) and Theorem 1, terms $I, I I, I I I$ and $I V$ tend to 0 as $N$ tends to $+\infty$. Since $|\boldsymbol{m}|=1$, terms $V$ and $V I$ containing the super-exchange interactions, tend to 0 because they are are bounded by:

$$
\begin{aligned}
C\left(\sum_{i=0}^{p-1} \sum_{k=0}^{N-1}\left\|\gamma \widetilde{\boldsymbol{m}}^{N} \wedge \boldsymbol{\nu}\right\|_{\mathrm{L}^{\infty}\left(\mathbb{R}^{+} ; \mathrm{L}^{2}\left(\Gamma_{i}^{N,+} ; \mathbb{R}^{3}\right)\right)}^{2}\right)^{\frac{1}{2}}\left(\sum_{i=0}^{p-1} \sum_{k=0}^{N-1}\left\|\gamma^{+} \phi-\gamma^{-} \phi\right\|_{\mathrm{L}^{1}\left(0, T ; \mathrm{L}^{2}\left(\Gamma_{i}^{N} ; \mathbb{R}^{3}\right)\right)}^{2}\right)^{\frac{1}{2}} & \leq \frac{C^{\prime}}{N^{\frac{1}{2}}}\left\|\frac{\partial \phi}{\partial x_{3}}\right\|_{\mathrm{L}^{1}\left(0, T ; \mathrm{L}^{2}\left(\Omega ; \mathbb{R}^{3}\right)\right)}
\end{aligned}
$$

Taking the limit in (6.4) yields $\iint_{Q_{T}^{N}} \frac{\partial\left(\widetilde{\boldsymbol{m}}^{N} \cdot \boldsymbol{e}_{3}\right)}{\partial t} \phi(\boldsymbol{x}, t) \mathrm{d} \boldsymbol{x} \mathrm{d} t=0$, for all $\phi$ in $\mathcal{C}^{\infty}(\overline{\Omega \times(0, T)})$. Moreover, $\widetilde{\boldsymbol{m}}^{\infty} \wedge \boldsymbol{e}_{3}=0$, thus $\widetilde{\boldsymbol{m}}^{\infty}$ does not depend on the time.

Looking at the proof, we notice that:

Remark 13. The results of the Theorem 12 are still valid for $\rho<1$.

6.1.2. The case of $\rho=1$

This subcase is very similar to the previous one. But in this subcase super-exchange is not strong enough to force the independence of the two-scale limit with respect to the fast variable $y$. As in the previous subcase, we provide the reader with one example of a sequence of initial conditions. We use the same basic ideas: $\boldsymbol{m}_{0}^{N}$ constant in each subcell and equal to either $\boldsymbol{e}_{3}$ or $-\boldsymbol{e}_{3}$. Since super-exchange has been weakened with respect to the previous subcase, the number of sign change between the layers does not need to remain bounded independently of $n$.

We state and prove the following theorem:

Theorem 14. Let $\varpi=0$ and $\rho=1$. Let $\boldsymbol{m}_{0}^{N}$ be a sequence of initial conditions in $\mathrm{H}^{1}\left(\Omega^{N} ; \mathbb{R}^{3}\right)$, $\left|\boldsymbol{m}_{0}^{N}\right|=1$ a.e. in $\Omega^{N}$, satisfying the energy constraint (6.1). Let $\boldsymbol{m}^{N}$ be a sequence of weak solutions to the Landau-Lifshitz system in the sense of Definition 3. Let $\overline{\boldsymbol{m}}^{N}$ be the extension by 0 of $\boldsymbol{m}^{N}$ to $\Omega \times \mathbb{R}^{+}$. Then, up to a subsequence, $\overline{\boldsymbol{m}}^{N}$ two-scale converges to $\overline{\boldsymbol{m}}^{\infty}$. The limit $\overline{\boldsymbol{m}}^{\infty}$ is parallel to $\boldsymbol{e}_{3}$ a.e. in $\Omega \times(0, T) \times \widetilde{I}$. Furthermore, for almost all $\boldsymbol{x}, t, \overline{\boldsymbol{m}}^{\infty}(\boldsymbol{x}, t, \cdot)$ does not depend on time, is constant in each interval $\widetilde{I}_{i}, 0 \leq i \leq p-1$ and is null in $\widetilde{J}$.

Moreover, let $\overline{\boldsymbol{m}}_{0}^{N}$ be the extension by 0 of $\boldsymbol{m}_{0}^{N}$ outside $\Omega^{N}$. If there exists $\overline{\boldsymbol{m}}_{0}^{\infty} \overline{\text { in }} \mathrm{L}^{2}(\Omega \times Y)$ such that $\overline{\boldsymbol{m}}_{0}^{N}$ two-scale converges to $\boldsymbol{m}_{0}^{\infty}$ in $\mathrm{L}^{2}(\Omega \times Y)$, then the whole sequence converges and $\widetilde{\boldsymbol{m}}^{\infty}(\cdot, t, \cdot)=\boldsymbol{m}_{0}^{\infty}$ for all time $t \geq 0$ in $\Omega \times \widetilde{I}$.

Proof. Inequality (4.2d), and conditions (6.1) imply that

$$
\begin{aligned}
\left\|\boldsymbol{m}^{N}\right\|_{\mathrm{L}^{\infty}\left(\mathbb{R}^{+} ; \mathrm{H}^{1}\left(\Omega^{N} ; \mathbb{R}^{3}\right)\right)}^{2} & \leq C,
\end{aligned} \quad\left\|\frac{\partial \boldsymbol{m}^{N}}{\partial t}\right\|_{\mathrm{L}^{2}\left(\Omega^{N} \times \mathbb{R}^{+} ; \mathbb{R}^{3}\right)}^{2} \leq C,
$$


According to Proposition 23 in the appendix, there exists an extension of $\widetilde{\boldsymbol{m}}^{N}$ satisfying

$$
\begin{aligned}
\left\|\widetilde{\boldsymbol{m}}^{N}\right\|_{L^{\infty}\left(\Omega \times \mathbb{R}^{+}\right)} & \leq 1, & \left\|\frac{\partial \widetilde{\boldsymbol{m}}^{N}}{\partial t}\right\|_{L^{2}\left(\Omega \times \mathbb{R}^{+} ; \mathbb{R}^{3}\right)} \leq C, \\
\left\|\frac{\partial \widetilde{\boldsymbol{m}}^{N}}{\partial x_{1}}\right\|_{L^{\infty}\left(\mathbb{R}^{+} ; L^{2}\left(\Omega ; \mathbb{R}^{3}\right)\right)} & \leq C, & \left\|\frac{\partial \widetilde{\boldsymbol{m}}^{N}}{\partial x_{2}}\right\|_{L^{\infty}\left(\mathbb{R}^{+} ; L^{2}\left(\Omega ; \mathbb{R}^{3}\right)\right)} \leq C, \\
\left\|\frac{\partial \widetilde{\boldsymbol{m}}^{N}}{\partial x_{3}}\right\|_{L^{\infty}\left(\mathbb{R}^{+} ; \mathrm{L}^{2}\left(\Omega ; \mathbb{R}^{3}\right)\right)} & \leq C N . &
\end{aligned}
$$

There exists $\widetilde{\boldsymbol{m}}^{\infty}$ in $\mathrm{L}_{\text {loc }}^{2}\left(\Omega \times \mathbb{R}^{+} \times(0,1)\right)$ two-scale limit of a subsequence $\widetilde{\boldsymbol{m}}^{N_{k}}$, i.e.,

$$
\lim _{k \rightarrow \infty} \iint_{\Omega \times(0, T)} \widetilde{\boldsymbol{m}}^{N_{k}}(\boldsymbol{x}, t) \phi\left(\boldsymbol{x}, t, \frac{N_{k}}{L} x_{3}\right) \mathrm{d} t \mathrm{~d} \boldsymbol{x}=\iint_{\Omega \times(0, T)} \int_{Y} \widetilde{\boldsymbol{m}}^{\infty}(\boldsymbol{x}, t, y) \phi(\boldsymbol{x}, t, y) \mathrm{d} t \mathrm{~d} y \mathrm{~d} \boldsymbol{x},
$$

for all time $T>0$ and for all $\phi$ in $\mathcal{C}^{\infty}(\overline{\Omega \times(0, T)}) \otimes \mathcal{C}_{\#}^{\infty}(Y)$.

We need to establish the characteristics of $\widetilde{\boldsymbol{m}}^{\infty}$. We begin by proving a simple lemma:

Lemma 15. For all $\boldsymbol{x}, t$ in $\Omega \times \mathbb{R}^{+}$, the function $\widetilde{\boldsymbol{m}}^{\infty}(\boldsymbol{x}, t, \cdot)$ is constant on each interval $I_{i}, i$ in $\mathbb{N}, 0 \leq i \leq p-1$.

Proof of the lemma. Let $T>0$ and $\phi(\boldsymbol{x}, t, y)=\psi(\boldsymbol{x}, t) \zeta(y)$ where $\zeta$ has a compact support included in $\widetilde{I}$. Then,

$$
\begin{aligned}
\iint_{\Omega \times(0, T)} \frac{\partial \widetilde{\boldsymbol{m}}^{N}}{\partial x_{3}}(\boldsymbol{x}, t) \psi(\boldsymbol{x}, t) \zeta\left(\frac{N}{L} \boldsymbol{x}\right) \mathrm{d} \boldsymbol{x} \mathrm{d} t & \\
=-\iint_{\Omega \times(0, T)} \widetilde{\boldsymbol{m}}^{N}(\boldsymbol{x}) \frac{\partial \psi}{\partial x_{3}}(\boldsymbol{x}, t) \zeta\left(\frac{N}{L} x_{3}\right) \mathrm{d} \boldsymbol{x} \mathrm{d} t & \quad-\frac{N}{L} \iint_{\Omega \times(0, T)} \widetilde{\boldsymbol{m}}^{N}(\boldsymbol{x}, t) \psi(\boldsymbol{x}, t) \zeta^{\prime}\left(\frac{N}{L} x_{3}\right) \mathrm{d} \boldsymbol{x} \mathrm{d} t .
\end{aligned}
$$

We divide this equality by $N$ and take the limit in each term as $N$ tends to $+\infty$, using the properties of two-scale convergence. We obtain

$$
\iint_{\Omega \times(0, T)} \psi(\boldsymbol{x}, t) \int_{\widetilde{I}} \widetilde{\boldsymbol{m}}^{\infty}(\boldsymbol{x}, t, y) \zeta^{\prime}(y) \mathrm{d} y \mathrm{~d} \boldsymbol{x}=0,
$$

for all $\zeta$ in $\mathcal{C}_{c}^{\infty}(\widetilde{I})$, for all $\psi$ in $\mathcal{C}_{c}^{\infty}(\Omega \times(0, T))$, and for all finite time $T>0$.

Let $\widetilde{\boldsymbol{m}}_{i}^{\infty}(\boldsymbol{x}, t)$ be the value of the function $\widetilde{\boldsymbol{m}}^{\infty}(\boldsymbol{x}, t, \cdot)$ on the interval $\widetilde{I}_{i}$. Since the two quantities $\| \boldsymbol{m}^{N} \wedge$ $\boldsymbol{e}_{3} \|_{\mathrm{L}^{2}\left(\Gamma^{N,+} \cup \Gamma^{N,-}\right)}^{2}$ and $\left\|\frac{\partial \boldsymbol{m}^{N}}{\partial x_{3}}\right\|_{\mathrm{L}^{2}\left(\Omega^{N}\right)}^{2}$ are bounded by $\mathrm{E}^{N}\left(\boldsymbol{m}_{0}^{N}\right)$, Lemma 11 and inequality (4.2d) yield that:

$$
\left\|\boldsymbol{m}^{N} \wedge \boldsymbol{e}_{3}\right\|_{\mathrm{L}^{\infty}\left(\mathbb{R}^{+} ; \mathrm{L}^{2}\left(\Omega^{N} ; \mathbb{R}^{3}\right)\right)}^{2} \leq \frac{C \mathrm{E}^{N}\left(\boldsymbol{m}_{0}^{N}\right)}{N},
$$

for some constant $C>0$. Since the sequence of initial conditions satisfies the energy boundedness (6.1), there exists $C^{\prime}>0$, independent of $N$, such that

$$
\left\|\boldsymbol{m}^{N} \wedge \boldsymbol{e}_{3}\right\|_{L^{\infty}\left(\mathbb{R}^{+} ; L^{2}\left(\Omega^{N} ; \mathbb{R}^{3}\right)\right)} \leq \frac{C^{\prime}}{\sqrt{N}}
$$

Therefore, $\widetilde{\boldsymbol{m}}^{\infty} \wedge \boldsymbol{e}_{3}=0$ a.e. in $\Omega \times \mathbb{R}^{+} \times \widetilde{I}$. 
Let $T>0$ be a finite time. Let $\phi(x, t)=\phi\left(\boldsymbol{x}, t, \frac{N}{L} x_{3}\right) \boldsymbol{e}_{3}$, with $\phi$ belonging to $\mathcal{C}^{\infty}(\overline{\Omega \times(0, T)}) \otimes \mathcal{C}_{\#}^{\infty}(Y)$, as the test function in $(4.2 \mathrm{~b})$, such that $\phi(\boldsymbol{x}, t, \cdot)$ is constant in each interval $\widetilde{I}_{i}$. Let $\phi_{i}(\boldsymbol{x}, t)$ be the value of $\phi(\boldsymbol{x}, t, \cdot)$ on the interval $\widetilde{I}_{i}$. We obtain

$$
\begin{gathered}
\sum_{i=0}^{p-1} \iint_{\Omega_{i}^{N} \times(0, T)} \frac{\partial\left(\widetilde{\boldsymbol{m}}^{N} \cdot \boldsymbol{e}_{3}\right)}{\partial t} \phi_{i} \mathrm{~d} \boldsymbol{x} \mathrm{d} t=\alpha \sum_{i=0}^{p-1} \iint_{\Omega_{i}^{N} \times(0, T)}\left(\widetilde{\boldsymbol{m}}^{N} \wedge \boldsymbol{e}_{3}\right) \cdot \frac{\partial \widetilde{\boldsymbol{m}}^{N}}{\partial t} \phi_{i} \mathrm{~d} \boldsymbol{x} \mathrm{d} t \\
-\left(1+\alpha^{2}\right) \sum_{i=0}^{p-1} \sum_{j=1}^{3} A_{i} \iint_{\Omega_{i}^{N} \times(0, T)}\left(\widetilde{\boldsymbol{m}}^{N} \wedge \boldsymbol{e}_{3}\right) \cdot \frac{\partial \widetilde{\boldsymbol{m}}^{N}}{\partial x_{j}} \frac{\partial \phi_{i}}{\partial x_{j}} \mathrm{~d} \boldsymbol{x} \mathrm{d} t \\
+\left(1+\alpha^{2}\right) \sum_{i=0}^{p-1} \iint_{\Omega_{i}^{N} \times(0, T)}\left(\widetilde{\boldsymbol{m}}^{N} \wedge \boldsymbol{e}_{3}\right) \cdot \mathcal{H}_{d}\left(\widetilde{\boldsymbol{m}}^{N}\right) \phi_{i} \mathrm{~d} \boldsymbol{x} \mathrm{d} t \\
-\left(1+\alpha^{2}\right) \sum_{i=0}^{p-1} \iint_{\Omega_{i}^{N} \times(0, T)}\left(\widetilde{\boldsymbol{m}}^{N} \wedge \boldsymbol{e}_{3}\right) \cdot \mathbf{K}_{i} \widetilde{\boldsymbol{m}}^{N} \phi_{i} \mathrm{~d} \boldsymbol{x} \mathrm{d} t \\
+\left(1+\alpha^{2}\right) \sum_{i=0}^{p-1} \frac{J_{1, i}}{N} \iint_{\Gamma_{i}^{N} \times(0, T)}\left(\gamma^{+} \widetilde{\boldsymbol{m}}^{N} \wedge \boldsymbol{e}_{3}\right) \cdot \gamma^{-} \widetilde{\boldsymbol{m}}^{N}\left(\gamma^{+} \phi_{i}-\gamma^{-} \phi_{i-1}\right) \mathrm{d} \boldsymbol{x} \mathrm{d} t \\
+2\left(1+\alpha^{2}\right) \sum_{i=0}^{p-1} \frac{J_{2, i}}{N} \iint_{\Gamma_{i}^{N} \times(0, T)}\left(\gamma^{+} \widetilde{\boldsymbol{m}}^{N} \cdot \gamma^{-} \widetilde{\boldsymbol{m}}^{N}\right)\left(\gamma^{+} \widetilde{\boldsymbol{m}}^{N} \wedge \boldsymbol{e}_{3}\right) \cdot \gamma^{-} \widetilde{\boldsymbol{m}}^{N}\left(\gamma^{+} \phi_{i}-\gamma^{-} \phi_{i-1}\right) \mathrm{d} \boldsymbol{x} \mathrm{d} t .
\end{gathered}
$$

As in the previous section, the first four terms of the right hand-side tend to 0 . The last two terms are bounded by

$$
\begin{aligned}
& \frac{C}{N^{\frac{1}{2}}}\left(\sum_{i=0}^{p-1} \sum_{k=0}^{N-1}\left\|\gamma \boldsymbol{m}^{N} \wedge \boldsymbol{\nu}\right\|_{\mathrm{L}^{\infty}\left(\mathbb{R}^{+} ; \mathrm{L}^{2}\left(\Gamma_{i, k}^{N,+} ; \mathbb{R}^{3}\right)\right)}^{2}\right)^{\frac{1}{2}} \\
& \quad \times\left(\frac{1}{N} \sum_{i=0}^{p-1} \sum_{k=0}^{N-1}\left\|\gamma^{+} \phi_{i+1}-\gamma^{-} \phi_{i}\right\|_{\mathrm{L}^{1}\left(0, T ; \mathrm{L}^{2}\left(\Gamma_{i, k}^{N} ; \mathbb{R}^{3}\right)\right)}^{2}\right)^{\frac{1}{2}} \leq \frac{C^{\prime}}{N^{\frac{1}{2}}}\|\phi\|_{\mathrm{L}^{1}\left(0, T ; \mathrm{L}^{2}(\Omega \times Y)\right)},
\end{aligned}
$$

and also converge to 0 . Thus, taking the limit in equation (6.6), when $N$ tend to $+\infty$, we obtain:

$$
\frac{\partial \widetilde{\boldsymbol{m}}_{i}^{\infty}}{\partial t}=0
$$

for all integers $i, 0 \leq i \leq p-1$. The limit $\widetilde{\boldsymbol{m}}^{\infty}(\boldsymbol{x}, t, y)$ depends only on the slow variable $\boldsymbol{x}$ and on the sublayer $\widetilde{I}_{i}$ to which $y$ belongs. Since $\overline{\boldsymbol{m}}^{N}=\chi^{N} \widetilde{\boldsymbol{m}}^{N}$, we can deduce the convergence of a subsequence of $\boldsymbol{m}^{-N}$ to $\overline{\boldsymbol{m}}^{\infty}=\chi \widetilde{\boldsymbol{m}}^{N}$.

Remark 16. In this case, super-exchange is not sufficiently strong to force the non variation of $\widetilde{\boldsymbol{m}}^{\infty}$ across the $p$ sublayers.

Looking at the proof, we notice that:

Remark 17. The results of Theorem 14 are still valid for $\rho \geq 1$.

\subsubsection{Physical interpretation}

When surface anisotropy dominates all other terms, the limit of the magnetization, as the number of layer $N$ tends to $+\infty$, is stationary and aligns itself parallel to the normal of the layers. The magnetization of a 
multilayer is extremely rigid and the energy necessary to change the magnetization becomes infinite as the number of layers tends to $+\infty$. A physical analysis of the reversal of magnetization in multilayers can be consulted in [10].

\subsection{Strong super-exchange}

In this section, we study the homogenization of the Landau-Lifshitz equation when the magnitude of surface anisotropy is proportional to the interlayer distance. We assume that the exponent $\varpi=1$. In this case, we can only obtain results if the super-exchange exponent $\rho$ is equal to -1 , thus having the magnitude of the super-exchange interaction vary proportionally to the number of layers $N$. Only in that case, do we have the necessary compactness to take the limit in nonlinear terms. When $\rho=-1$, we have a "good" extension operator by Proposition ${ }^{4} 23$ in the appendix, and nonlinear terms converge by Theorem 7 .

Before stating the theorem, we provide the reader with an example of sequence of initial conditions satisfying inequality (6.1). We may just choose $\boldsymbol{m}_{0}$ in $\mathrm{H}^{1}\left(\Omega ; \mathbb{R}^{3}\right)$ and let $\boldsymbol{m}_{0}^{N}$ be the restriction of $\boldsymbol{m}_{0}$ to $\Omega^{N}$.

Theorem 18. Let $\varpi=1$ and $\rho=-1$. Let $\boldsymbol{m}_{0}^{N}$ be a sequence of initial conditions in $\mathrm{H}^{1}\left(\Omega^{N} ; \mathbb{R}^{3}\right)$, $\left|\boldsymbol{m}_{0}^{N}\right|=1$ a.e. in $\Omega^{N}$, satisfying the energy constraint (6.1). Let $\boldsymbol{m}^{N}$ be a sequence of weak solutions to the Landau-Lifshitz system in the sense of Definition 3 . Then there exists a sequence of extensions to $\boldsymbol{m}^{N}$ in $\mathrm{H}_{\mathrm{loc}}^{1}\left(\Omega \times \mathbb{R}^{+} ; \mathbb{R}^{3}\right)$ denoted $\widetilde{\boldsymbol{m}}^{N}$ and a subsequence of $\widetilde{\boldsymbol{m}}^{N}$ that two-scale converges to $\widetilde{\boldsymbol{m}}^{\infty}$. This limit belongs to $\mathrm{L}^{\infty}\left(\mathbb{R}^{+} ; \mathrm{H}^{1}\left(\Omega^{N} ; \mathbb{R}^{3}\right)\right)$ and to $\mathrm{H}_{\mathrm{loc}}^{1}\left(\Omega \times \mathbb{R}^{+} ; \mathbb{R}^{3}\right)$ and satisfies variational equality $(6.31)$. Thus, $\widetilde{\boldsymbol{m}}^{\infty}$ is a weak solution to the homogenized system:

$$
\begin{aligned}
\frac{\partial \widetilde{\boldsymbol{m}}^{\infty}}{\partial t}-\alpha \widetilde{\boldsymbol{m}} \wedge \frac{\partial \widetilde{\boldsymbol{m}}^{\infty}}{\partial t}= & -\left(1+\alpha^{2}\right)\left(A_{T} \widetilde{\boldsymbol{m}}^{\infty} \wedge \triangle_{2 \mathrm{D}} \widetilde{\boldsymbol{m}}^{\infty}+A_{N} \widetilde{\boldsymbol{m}}^{\infty} \wedge \frac{\partial^{2} \widetilde{\boldsymbol{m}}^{\infty}}{\partial x_{3}^{2}}\right) \\
& +\left(1+\alpha^{2}\right) \widetilde{\boldsymbol{m}}^{\infty} \wedge \overline{\mathbf{K}} \widetilde{\boldsymbol{m}}^{\infty}-\left(1+\alpha^{2}\right) \bar{K}\left(\widetilde{\boldsymbol{m}}^{\infty} \cdot \boldsymbol{e}_{3}\right) \widetilde{\boldsymbol{m}}^{\infty} \wedge \boldsymbol{e}_{3} \\
& -\left(1+\alpha^{2}\right) \bar{\chi} \widetilde{\boldsymbol{m}}^{\infty} \wedge \mathcal{H}_{d}\left(\widetilde{\boldsymbol{m}}^{\infty}\right), \quad \text { in } \Omega \times \mathbb{R}^{+}, \\
\left|\widetilde{\boldsymbol{m}}^{\infty}\right|= & 1 \text { a.e. in } \Omega \times \mathbb{R}^{+}, \\
\widetilde{\boldsymbol{m}}^{\infty} \wedge \frac{\partial \widetilde{\boldsymbol{m}}}{\partial \boldsymbol{\nu}}= & 0, \text { on } \partial \Omega \times \mathbb{R}^{+},
\end{aligned}
$$

where

$$
\begin{aligned}
A_{T} & =\sum_{i=0}^{p-1} \frac{\bar{\chi}_{i}}{\bar{\chi}} A_{i}, & A_{N} & =\frac{1}{\bar{\chi}} \frac{1}{\sum_{i=0}^{p-1}\left(\frac{\bar{\chi}_{i}}{A_{i}}+\frac{1}{L\left(J_{1, i}+2 J_{2, i}\right)}\right)}, \\
\overline{\mathbf{K}} & =\sum_{i=0}^{p-1} \frac{\bar{\chi}_{i}}{\bar{\chi}} \mathbf{K}_{i}, & \bar{K} & =\sum_{i=0}^{p-1} \frac{K_{s, i}^{+}+K_{s, i}^{-}}{L \bar{\chi}}-(1-\bar{\chi}),
\end{aligned}
$$

and, denoting by $\boldsymbol{\nu}=\left(\nu_{1}, \nu_{2}, \nu_{3}\right)$ the exterior normal,

$$
\frac{\partial \widetilde{\boldsymbol{m}}}{\partial \boldsymbol{\nu}_{A}}=A_{T} \nu_{1} \frac{\partial \widetilde{\boldsymbol{m}}}{\partial x_{1}}+A_{T} \nu_{2} \frac{\partial \widetilde{\boldsymbol{m}}}{\partial x_{2}}+A_{N} \nu_{3} \frac{\partial \widetilde{\boldsymbol{m}}}{\partial x_{3}} .
$$

Moreover, let $\overline{\boldsymbol{m}}_{0}^{N}$ be the extension by 0 of $\boldsymbol{m}_{0}^{N}$ outside $\Omega^{N}$. If $\overline{\boldsymbol{m}}_{0}^{N}$ tends weakly to $\bar{\chi} \boldsymbol{m}_{0}^{\infty}$ in $\mathrm{L}^{2}\left(\Omega ; \mathbb{R}^{3}\right)$, then:

$$
\widetilde{\boldsymbol{m}}^{\infty}(\cdot, 0)=\boldsymbol{m}_{0}^{\infty} \quad \text { in } \Omega
$$

\footnotetext{
${ }^{4}$ In Hamdache [9], it is asserted, Lemma 3.2, that the extension operators, with injection constants not depending on $N$, exist when the magnitude of the super-exchange interaction does not depend on the interlayer distance, i.e. when $\rho=0$. This is incorrect: see the counter-example in Section A.2.
} 
Proof. Before proving the theorem, we make the following remark:

Remark 19. As the solutions to the Landau-Lifshitz system are (most probably) not unique, see the nonuniqueness result in Alouges and Soyeur [4], it cannot be deduced that the whole sequence converges in Theorem 18.

By the energy inequality (4.2d) and the conditions (6.1), there exists a constant $C>0$, such that

$$
\begin{aligned}
\left\|\boldsymbol{m}^{N}\right\|_{L^{\infty}\left(\Omega^{N} \times \mathbb{R}^{+} ; \mathbb{R}^{3}\right)} & =1, & \left\|\nabla \boldsymbol{m}^{N}\right\|_{L^{\infty}\left(\mathbb{R}^{+} ; \mathrm{L}^{2}\left(\Omega^{N} ; \mathbb{R}^{3}\right)\right)} & \leq C \\
\left\|\frac{\partial \boldsymbol{m}^{N}}{\partial t}\right\|_{L^{2}\left(\Omega^{N} \times \mathbb{R}^{+} ; \mathbb{R}^{3}\right)} & \leq C, & N\left\|\gamma^{+} \boldsymbol{m}^{N}-\gamma^{-} \boldsymbol{m}^{N}\right\|_{L^{\infty}\left(\mathbb{R}^{+} ; \mathrm{L}^{2}\left(\Gamma^{N} ; \mathbb{R}^{3}\right)\right)}^{2} & \leq C .
\end{aligned}
$$

According to Proposition 23 in the appendix, there exists a constant $C>0$, such that for every integer $N$ in $\mathbb{N}^{*}$, there exists $\widetilde{\boldsymbol{m}}^{N}$ belonging to $\mathrm{L}^{\infty}\left(\mathbb{R}^{+} ; \mathrm{H}^{1}\left(\Omega ; \mathbb{R}^{3}\right)\right)$ and to $\mathrm{H}^{1}\left(\Omega \times(0, T) ; \mathbb{R}^{3}\right)$, such that

$$
\begin{aligned}
\left\|\widetilde{\boldsymbol{m}}^{N}\right\|_{\mathrm{L}^{\infty}\left(\Omega \times \mathbb{R}^{+} ; \mathbb{R}^{3}\right)} & \leq 1, & \left\|\nabla \widetilde{\boldsymbol{m}}^{N}\right\|_{\mathrm{L}^{\infty}\left(\mathbb{R}^{+} ; \mathrm{L}^{2}\left(\Omega ; \mathbb{R}^{3}\right)\right)} \leq C, \\
\left\|\frac{\partial \widetilde{\boldsymbol{m}}^{N}}{\partial t}\right\|_{L^{2}\left(\Omega \times \mathbb{R}^{+} ; \mathbb{R}^{3}\right)} & \leq C, & \frac{1}{N}\left\|N\left(\gamma^{+} \widetilde{\boldsymbol{m}}^{N}-\gamma^{-} \widetilde{\boldsymbol{m}}^{N}\right)\right\|_{\mathrm{L}^{\infty}\left(\mathbb{R}^{+} ; \mathrm{L}^{2}\left(\Gamma^{N} ; \mathbb{R}^{3}\right)\right)}^{2} \leq C .
\end{aligned}
$$

By Theorems 6 and 8, and Proposition 1.14 in [2], there exist

$$
\begin{aligned}
& \widetilde{\boldsymbol{m}}^{\infty} \text { in } \mathrm{L}^{\infty}\left(\mathbb{R}^{+} ; \mathrm{H}^{1}\left(\Omega ; \mathbb{R}^{3}\right)\right) \cap \mathrm{H}^{1}\left(\Omega \times(0, T) ; \mathbb{R}^{3}\right), \\
& \widetilde{\boldsymbol{m}}_{1}^{\infty} \text { in } \mathrm{L}^{\infty}\left(\mathbb{R}^{+} ; \mathrm{L}^{2}\left(\Omega ; \mathrm{H}_{\#}^{1}\left(Y ; \mathbb{R}^{3}\right)\right),\right. \\
& \boldsymbol{w}_{i}^{\infty} \text { in } \mathrm{L}^{\infty}\left(\mathbb{R}^{+} ; \mathrm{L}^{2}\left(\Omega ; \mathbb{R}^{3}\right)\right), \quad 0 \leq i \leq p-1, \\
& \boldsymbol{h}^{\infty} \text { in } \mathrm{L}^{\infty}\left(\mathbb{R}^{+} ; \mathrm{L}_{\text {loc }}^{2}\left(\mathbb{R}^{3} ; \mathbb{R}^{3}\right)\right),
\end{aligned}
$$

such that, for all finite time $T>0$, for all $\phi$ in $\mathcal{C}^{\infty}\left(\overline{\Omega \times \mathbb{R}^{+}}\right) \otimes \mathcal{C}_{\#}^{\infty}(Y)$ :

$$
\begin{gathered}
\lim _{N \rightarrow \infty} \iint_{Q_{T}} \widetilde{\boldsymbol{m}}^{N}(\boldsymbol{x}, t) \phi\left(\boldsymbol{x}, t, \frac{N}{L} x_{3}\right) \mathrm{d} \boldsymbol{x} \mathrm{d} t=\iint_{Q_{T}} \widetilde{\boldsymbol{m}}^{\infty}(\boldsymbol{x}, t) \int_{Y} \phi(\boldsymbol{x}, t, y) \mathrm{d} y \mathrm{~d} \boldsymbol{x} \mathrm{d} t \\
\lim _{N \rightarrow \infty} \iint_{Q_{T}} \mathcal{H}_{d}\left(\chi^{N} \widetilde{\boldsymbol{m}}^{N}\right) \phi\left(\boldsymbol{x}, t, \frac{N}{L} x_{3}\right) \mathrm{d} \boldsymbol{x} \mathrm{d} t=\iint_{Q_{T}} \int_{Y} \boldsymbol{h}^{\infty}(\boldsymbol{x}, t, y) \phi(\boldsymbol{x}, t, y) \mathrm{d} y \mathrm{~d} \boldsymbol{x} \mathrm{d} t \\
\lim _{N \rightarrow \infty} \iint_{Q_{T}} \frac{\partial \widetilde{\boldsymbol{m}}^{N}(\boldsymbol{x})}{\partial x_{3}} \phi\left(\boldsymbol{x}, t, \frac{N}{L} x_{3}\right) \mathrm{d} \boldsymbol{x} \mathrm{d} t=\iint_{Q_{T}} \int_{Y}\left(\frac{\partial \widetilde{\boldsymbol{m}}^{\infty}}{\partial x_{3}}(\boldsymbol{x}, t)+\frac{\partial \widetilde{\boldsymbol{m}}_{1}^{\infty}}{\partial y}(\boldsymbol{x}, t, y)\right) \phi(\boldsymbol{x}, t, y) \mathrm{d} y \mathrm{~d} \boldsymbol{x} \mathrm{d} t \\
\lim _{N \rightarrow \infty} \frac{1}{N} \iint_{\Gamma^{N} \times(0, T)} \widetilde{\boldsymbol{m}}^{N}(\boldsymbol{x}) \phi\left(\boldsymbol{x}, t, \frac{N}{L} x_{3}\right) \mathrm{d} \sigma(\boldsymbol{x})=\frac{1}{L} \iint_{Q_{T}} \widetilde{\boldsymbol{m}}^{\infty}(\boldsymbol{x}) \sum_{i=0}^{p-1} \phi\left(\boldsymbol{x}, t, z_{i}\right) \mathrm{d} \boldsymbol{x} \mathrm{d} t \\
\lim _{N \rightarrow \infty} \iint_{\Gamma^{N} \times(0, T)}\left(\gamma^{+} \widetilde{\boldsymbol{m}}^{N}(\boldsymbol{x}, t)-\gamma^{-} \widetilde{\boldsymbol{m}}^{N}(\boldsymbol{x}, t)\right) \phi\left(\boldsymbol{x}, t, \frac{N}{L} x_{3}\right) \mathrm{d} \sigma(\boldsymbol{x}) \mathrm{d} t \\
=\frac{1}{L} \sum_{i=0}^{p-1} \iint_{Q_{T}} \boldsymbol{w}_{i}^{\infty}(\boldsymbol{x}, t) \phi\left(\boldsymbol{x}, t, z_{i}\right) \mathrm{d} \boldsymbol{x} \mathrm{d} t .
\end{gathered}
$$

The limit (6.11d) still holds if we replace $\Gamma^{N}$ by $\Gamma^{N,+}$ or $\Gamma^{N,-}$. 
First, we prove that $\left|\widetilde{\boldsymbol{m}}^{\infty}\right|=1$. Since $\widetilde{\boldsymbol{m}}^{N}$ is bounded in $\mathrm{H}^{1}(\Omega \times(0, T))$, up to a subsequence, $\widetilde{\boldsymbol{m}}^{N}$ converges strongly in $\mathrm{L}^{2}(\Omega \times(0, T))$ to $\widetilde{\boldsymbol{m}}^{\infty}$. But

$$
\left\|\left|\widetilde{\boldsymbol{m}}^{N}\right|-1\right\|_{\mathrm{L}^{2}(\Omega \times(0, T))}=\left\|\left|\widetilde{\boldsymbol{m}}^{N}\right|-1\right\|_{\mathrm{L}^{2}\left(\left(\Omega \backslash \Omega^{N}\right) \times(0, T)\right)} \leq \max \left(\theta_{i}\right) \frac{L}{N}\left\|\frac{\partial \widetilde{\boldsymbol{m}}^{N}}{\partial x_{3}}\right\|_{\mathrm{L}^{2}\left(\left(\Omega \backslash \Omega^{N}\right) \times(0, T)\right)}
$$

for all $T>0$. Therefore $\left|\widetilde{\boldsymbol{m}}^{\infty}\right|=1$.

Since $\boldsymbol{m}^{N}$ is a weak solution to the Landau-Lifshitz system, we have

$$
\begin{aligned}
& \iint_{Q_{T}^{N}} \frac{\partial \widetilde{\boldsymbol{m}}^{N}}{\partial t} \cdot \boldsymbol{\phi}^{N} \mathrm{~d} \boldsymbol{x} \mathrm{d} t-\alpha \iint_{Q_{T}^{N}}\left(\widetilde{\boldsymbol{m}}^{N} \wedge \frac{\partial \widetilde{\boldsymbol{m}}^{N}}{\partial t}\right) \cdot \boldsymbol{\phi}^{N} \mathrm{~d} \boldsymbol{x} \mathrm{d} t \\
&=\left(1+\alpha^{2}\right) \sum_{i=0}^{p-1} A_{i} \iint_{Q_{T, i}^{N}} \sum_{j=1}^{3}\left(\widetilde{\boldsymbol{m}}^{N} \wedge \frac{\partial \widetilde{\boldsymbol{m}}^{N}}{\partial x_{j}}\right) \cdot \frac{\partial \boldsymbol{\phi}}{\partial x_{j}}\left(\boldsymbol{x}, t, \frac{N}{L} x_{3}\right) \mathrm{d} \boldsymbol{x} \mathrm{d} t \\
&+\left(1+\alpha^{2}\right) \frac{N}{L} \sum_{i=0}^{p-1} A_{i} \iint_{Q_{T, i}^{N}}\left(\widetilde{\boldsymbol{m}}^{N} \wedge \frac{\partial \widetilde{\boldsymbol{m}}^{N}}{\partial x_{3}}\right) \cdot \frac{\partial \boldsymbol{\phi}}{\partial y}\left(\boldsymbol{x}, t, \frac{N}{L} x_{3}\right) \mathrm{d} \boldsymbol{x} \mathrm{d} t \\
&-\left(1+\alpha^{2}\right) \iint_{Q_{T}^{N}}\left(\widetilde{\boldsymbol{m}}^{N} \wedge\left(\mathcal{H}_{d}\left(\widetilde{\boldsymbol{m}}^{N}\right)-\mathbf{K}_{i} \widetilde{\boldsymbol{m}}^{N}\right)\right) \cdot \boldsymbol{\phi}^{N} \mathrm{~d} \boldsymbol{x} \mathrm{d} t \\
&-\left(1+\alpha^{2}\right) \sum_{i=0}^{p-1} \frac{K_{s, i}^{+}}{N} \iint_{\Gamma_{i}^{N,+} \times(0, T)}\left(\boldsymbol{\nu} \cdot \gamma \widetilde{\boldsymbol{m}}^{N}\right)\left(\gamma \widetilde{\boldsymbol{m}}^{N} \wedge \boldsymbol{\nu}\right) \cdot \gamma \boldsymbol{\phi}^{N} \mathrm{~d} \sigma(\boldsymbol{x}) \mathrm{d} t \\
&-\left(1+\alpha^{2}\right) \sum_{i=0}^{p-1} \frac{K_{s, i}^{-}}{N} \iint_{\Gamma_{i}^{N,-} \times(0, T)}\left(\boldsymbol{\nu} \cdot \gamma \widetilde{\boldsymbol{m}}^{N}\right)\left(\gamma \widetilde{\boldsymbol{m}}^{N} \wedge \boldsymbol{\nu}\right) \cdot \gamma \boldsymbol{\phi}^{N} \mathrm{~d} \sigma(\boldsymbol{x}) \mathrm{d} t \\
&-\left(1+\alpha^{2}\right) N \sum_{i=0}^{p-1}\left(J_{1, i} \iint_{\Gamma_{i}^{N} \times(0, T)}\left(\gamma^{+} \widetilde{\boldsymbol{m}}^{N} \wedge \gamma^{-} \widetilde{\boldsymbol{m}}^{N}\right) \cdot\left(\gamma^{+} \boldsymbol{\phi}^{N}-\gamma^{-} \boldsymbol{\phi}^{N}\right) \mathrm{d} \sigma(\boldsymbol{x}) \mathrm{d} t\right. \\
&+\left.2 J_{2, i} \iint_{\Gamma_{i}^{N} \times(0, T)}\left(\gamma^{+} \widetilde{\boldsymbol{m}}^{N} \cdot \gamma^{-} \widetilde{\boldsymbol{m}}^{N}\right)\left(\gamma^{+} \widetilde{\boldsymbol{m}}^{N} \wedge \gamma^{-} \widetilde{\boldsymbol{m}}^{N}\right) \cdot\left(\gamma^{+} \boldsymbol{\phi}^{N}-\gamma^{-} \boldsymbol{\phi}^{N}\right) \mathrm{d} \sigma(\boldsymbol{x}) \mathrm{d} t\right),
\end{aligned}
$$

for all $T>0$, for all $\phi$ in $\mathcal{C}^{\infty}\left(\overline{\Omega \times \mathbb{R}^{+}}\right) \otimes \mathcal{C}^{\infty}\left(\widetilde{I} ; \mathbb{R}^{3}\right)$, if $\phi^{N}(\boldsymbol{x}, t)=\phi\left(\boldsymbol{x}, t, \frac{N}{L} x_{3}\right)$. Let $\phi$ be independent of the fast variable $y$ and take the limit in equation (6.12). According to Theorems 7 and 10, we can take the limit in products. Thus,

$$
\begin{aligned}
\bar{\chi} \iint_{Q_{T}} \frac{\partial \widetilde{\boldsymbol{m}}^{\infty}}{\partial t} \cdot \boldsymbol{\phi} \mathrm{d} \boldsymbol{x} \mathrm{d} t-\bar{\chi} \alpha \iint_{Q_{T}}\left(\widetilde{\boldsymbol{m}}^{\infty} \wedge \frac{\partial \widetilde{\boldsymbol{m}}^{\infty}}{\partial t}\right) \cdot \boldsymbol{\phi} \mathrm{d} \boldsymbol{x} \mathrm{d} t \\
=\left(1+\alpha^{2}\right) \sum_{i=0}^{p-1} A_{i} \iint_{Q_{T}} \sum_{j=1}^{2}\left(\widetilde{\boldsymbol{m}}^{\infty} \wedge \frac{\partial \widetilde{\boldsymbol{m}}^{\infty}}{\partial x_{j}}\right) \cdot \frac{\partial \boldsymbol{\phi}}{\partial x_{j}} \mathrm{~d} \boldsymbol{x} \mathrm{d} t \\
+\left(1+\alpha^{2}\right) \sum_{i=0}^{p-1} A_{i} \bar{\chi}_{i} \iint_{Q_{T}}\left(\widetilde{\boldsymbol{m}}^{\infty} \wedge \int_{I_{i}}\left(\frac{\partial \widetilde{\boldsymbol{m}}^{\infty}}{\partial x_{3}}+\frac{\partial \widetilde{\boldsymbol{m}}_{1}^{\infty}}{\partial y}\right) \mathrm{d} y\right) \cdot \frac{\partial \boldsymbol{\phi}}{\partial x_{3}} \mathrm{~d} \boldsymbol{x} \mathrm{d} t \\
+\left(1+\alpha^{2}\right) \sum_{i=0}^{p-1} \iint_{Q_{T}}\left(\widetilde{\boldsymbol{m}}^{\infty} \wedge \bar{\chi}_{i} \mathbf{K}_{i} \widetilde{\boldsymbol{m}}^{\infty}\right) \boldsymbol{\phi} \mathrm{d} \boldsymbol{x} \mathrm{d} t \\
-\left(1+\alpha^{2}\right) \iint_{Q_{T}}\left(\widetilde{\boldsymbol{m}}^{\infty} \wedge \int_{I} \boldsymbol{h}^{\infty} \mathrm{d} y\right) \boldsymbol{\phi} \mathrm{d} \boldsymbol{x} \mathrm{d} t
\end{aligned}
$$




$$
\begin{aligned}
-\left(1+\alpha^{2}\right) \sum_{i=0}^{p-1} \frac{K_{s, i}^{+}+K_{s, i}^{-}}{L} \iint_{Q_{T}}\left(\boldsymbol{e}_{3} \cdot \widetilde{\boldsymbol{m}}^{\infty}\right)\left(\widetilde{\boldsymbol{m}}^{\infty} \wedge \boldsymbol{e}_{3}\right) \cdot \boldsymbol{\phi} \mathrm{d} \boldsymbol{x} \mathrm{d} t \\
\quad+\left(1+\alpha^{2}\right) \sum_{i=0}^{p-1}\left(J_{1, i}+2 J_{2, i}\right) \theta_{i} \iint_{Q_{T}}\left(\widetilde{\boldsymbol{m}}^{\infty} \wedge \boldsymbol{w}_{i}^{\infty}\right) \cdot \frac{\partial \boldsymbol{\phi}}{\partial x_{3}} \mathrm{~d} \boldsymbol{x} \mathrm{d} t
\end{aligned}
$$

for all $T>0$ and for all $\phi$ in $\mathcal{C}^{\infty}\left(\overline{\Omega \times(0, T)} ; \mathbb{R}^{3}\right)$. To obtain the equation satisfied by $\widetilde{\boldsymbol{m}}^{\infty}$, we only need to establish relations between $\widetilde{\boldsymbol{m}}^{\infty}, \widetilde{\boldsymbol{m}}_{1}^{\infty}, \boldsymbol{w}^{\infty}$ and $\boldsymbol{h}^{\infty}$. These are achieved in Lemma 20, and Propositions 21 and 22 .

We begin by proving a relation between $\boldsymbol{w}^{\infty}, \widetilde{\boldsymbol{m}}^{\infty}$ and $\widetilde{\boldsymbol{m}}_{1}^{\infty}$ that holds independently of the Landau-Lifshitz equation.

Lemma 20. For all $\boldsymbol{x}, t$ in $\Omega \times \mathbb{R}^{+}$,

$$
\int_{I} \frac{\partial \widetilde{\boldsymbol{m}}_{1}^{\infty}}{\partial y}(\boldsymbol{x}, t, y) \mathrm{d} y+\frac{1}{L} \sum_{i=0}^{p-1} \boldsymbol{w}_{i}^{\infty}(\boldsymbol{x}, t)=(1-\bar{\chi}) \frac{\partial \widetilde{\boldsymbol{m}}^{\infty}}{\partial x_{3}}(\boldsymbol{x}, t)
$$

Proof of the lemma. We have

$$
\begin{aligned}
\iint_{Q_{T}^{N}} \frac{\partial \widetilde{\boldsymbol{m}}^{N}}{\partial x_{3}}(\boldsymbol{x}, t) \phi\left(\boldsymbol{x}, t, \frac{N}{L} x_{3}\right) \mathrm{d} \boldsymbol{x} \mathrm{d} t & +\iint_{Q_{T}^{N}} \widetilde{\boldsymbol{m}}^{N}(\boldsymbol{x}, t) \frac{\partial \phi}{\partial x_{3}}\left(\boldsymbol{x}, t, \frac{N}{L} x_{3}\right) \mathrm{d} \boldsymbol{x} \mathrm{d} t \\
+\frac{N}{L} \iint_{Q_{T}^{N}} \widetilde{\boldsymbol{m}}^{N}(\boldsymbol{x}, t) \frac{\partial \phi}{\partial y}\left(\boldsymbol{x}, t, \frac{N}{L} x_{3}\right) \mathrm{d} \boldsymbol{x} \mathrm{d} t & \\
& =\sum_{i=0}^{p-1} \iint_{\Gamma_{i}^{N} \times(0, T)}\left(\gamma^{-} \widetilde{\boldsymbol{m}}^{N} \gamma^{-} \phi^{N}-\gamma^{+} \widetilde{\boldsymbol{m}}^{N} \gamma^{+} \phi^{N}\right) \mathrm{d} \sigma(\boldsymbol{x}) \mathrm{d} t
\end{aligned}
$$

for all $T>0$, for all $\phi$ in $\mathcal{C}_{c}^{\infty}(\Omega \times(0, T)) \otimes \mathcal{C}_{\#}^{\infty}(Y)$, where $\phi^{N}(\boldsymbol{x}, t)=\phi\left(\boldsymbol{x}, t, \frac{N}{L} x_{3}\right)$. Dividing equation (6.15) by $N$ and taking the limit does not yield any interesting result. Instead, we choose $\phi$ independent of the fast variable $y$. Then, we notice that

$$
\begin{aligned}
& \sum_{i=0}^{p-1} \iint_{\Gamma_{i}^{N} \times(0, T)} \gamma^{+} \widetilde{\boldsymbol{m}}^{N} \gamma^{+} \phi-\gamma^{-} \widetilde{\boldsymbol{m}}^{N} \gamma^{-} \phi \mathrm{d} \sigma(\boldsymbol{x}) \mathrm{d} t \\
&=\sum_{i=0}^{p-1} \iint_{\Gamma_{i}^{N} \times(0, T)}\left(\gamma^{+} \widetilde{\boldsymbol{m}}^{N}-\gamma^{-} \widetilde{\boldsymbol{m}}^{N}\right)\left(\frac{\gamma^{+} \phi+\gamma^{-} \phi}{2}\right) \mathrm{d} \sigma(\boldsymbol{x}) \mathrm{d} t \\
& \quad+\sum_{i=0}^{p-1} \iint_{\Gamma_{i}^{N} \times(0, T)}\left(\frac{\gamma^{+} \widetilde{\boldsymbol{m}}^{N}+\gamma^{-} \widetilde{\boldsymbol{m}}^{N}}{2}\right)\left(\gamma^{+} \phi-\gamma^{-} \phi\right) \mathrm{d} \sigma(\boldsymbol{x}) \mathrm{d} t .
\end{aligned}
$$

Taking the two-scale limit, by Taylor expansion of $\phi$, yields

$$
\frac{1}{L} \sum_{i=0}^{p-1} \iint_{\Omega \times(0, T)} \boldsymbol{w}_{i}^{\infty}(\boldsymbol{x}, t) \phi(\boldsymbol{x}, t) \mathrm{d} \boldsymbol{x} \mathrm{d} t+\sum_{i=0}^{p-1} \theta_{i} \iint_{\Omega \times(0, T)} \widetilde{\boldsymbol{m}}^{\infty}(\boldsymbol{x}, t) \frac{\partial \phi}{\partial x_{3}}(\boldsymbol{x}, t) \mathrm{d} \boldsymbol{x} \mathrm{d} t .
$$


Thus, if we take the limit in (6.15) as $N$ tends to $+\infty$, we obtain

$$
\begin{aligned}
\iint_{\Omega \times(0, T)} \int_{I}\left(\frac{\partial \widetilde{\boldsymbol{m}}^{\infty}}{\partial x_{3}}(\boldsymbol{x}, t)+\frac{\partial \widetilde{\boldsymbol{m}}_{1}^{\infty}}{\partial y}(\boldsymbol{x}, t, y)\right) \phi(\boldsymbol{x}, t) \mathrm{d} y \mathrm{~d} \boldsymbol{x} \mathrm{d} t \\
+\bar{\chi} \iint_{\Omega \times(0, T)} \widetilde{\boldsymbol{m}}^{\infty}(\boldsymbol{x}, t) \frac{\partial \phi}{\partial x_{3}}(\boldsymbol{x}, t) \mathrm{d} \boldsymbol{x} \mathrm{d} t \\
+\frac{1}{L} \sum_{i=0}^{p-1} \iint_{\Omega \times(0, T)} \boldsymbol{w}_{i}^{\infty}(\boldsymbol{x}, t) \phi(\boldsymbol{x}, t) \mathrm{d} \boldsymbol{x} \mathrm{d} t \\
+(1-\bar{\chi}) \iint_{\Omega \times(0, T)} \widetilde{\boldsymbol{m}}^{\infty}(\boldsymbol{x}, t) \frac{\partial \phi}{\partial x_{3}}(\boldsymbol{x}, t) \mathrm{d} \boldsymbol{x} \mathrm{d} t=0,
\end{aligned}
$$

for all time $T>0$, for all $\phi$ in $\mathcal{C}_{c}^{\infty}(\Omega \times(0, T))$.

The following proposition shows that we can express $\widetilde{\boldsymbol{m}}_{1}^{\infty}$ and $\boldsymbol{w}_{i}^{\infty}$ as functions of $\frac{\partial \widetilde{\boldsymbol{m}}^{\infty}}{\partial x_{3}}$.

Proposition 21. The function $\frac{\partial \widetilde{\boldsymbol{m}}_{1}^{\infty}}{\partial y}(\boldsymbol{x}, t, \cdot)$ is constant on each interval $I_{i}, 0 \leq i \leq p-1$. We denote by $\boldsymbol{v}_{i}^{\infty}$ the value of $\frac{\partial \widetilde{\boldsymbol{m}}_{1}^{\infty}}{\partial y}(\boldsymbol{x}, t, \cdot)$ when $y$ is in $I_{i}$. For every integer $i, 0 \leq i \leq p-1$,

$$
\begin{aligned}
A_{i}\left(\frac{\partial \widetilde{\boldsymbol{m}}^{\infty}}{\partial x_{3}}(\boldsymbol{x}, t)+\boldsymbol{v}_{i}^{\infty}(\boldsymbol{x}, t)\right) & =\frac{\frac{\partial \widetilde{\boldsymbol{m}}^{\infty}}{\partial x_{3}}(\boldsymbol{x}, t)}{\sum_{i=0}^{p-1}\left(\frac{\bar{\chi}_{i}}{A_{i}}+\frac{1}{L\left(J_{1, i}+2 J_{2, i}\right)}\right)}, \\
\left(J_{1, i}+2 J_{2, i}\right) \boldsymbol{w}_{i}^{\infty}(\boldsymbol{x}, t) & =\frac{\frac{\partial \widetilde{\boldsymbol{m}}^{\infty}}{\partial x_{3}}(\boldsymbol{x}, t)}{\sum_{i=0}^{p-1}\left(\frac{\bar{\chi}_{i}}{A_{i}}+\frac{1}{L\left(J_{1, i}+2 J_{2, i}\right)}\right)} .
\end{aligned}
$$

Proof of the proposition. We divide equation (6.12) by $N$ and take the limit:

$$
\begin{aligned}
\sum_{i=0}^{p-1} \iint_{\Omega \times(0, T)} \int_{I_{i}}\left(\widetilde{\boldsymbol{m}}^{\infty} \wedge\left(\frac{\partial \widetilde{\boldsymbol{m}}^{\infty}}{\partial x_{3}}(\boldsymbol{x}, t)+\frac{\partial \widetilde{\boldsymbol{m}}_{1}^{\infty}}{\partial y}(\boldsymbol{x}, t, y)\right)\right) \cdot \frac{\partial \phi}{\partial y}(\boldsymbol{x}, t, y) \mathrm{d} \boldsymbol{x} \mathrm{d} t \\
\quad+\sum_{i=0}^{p-1} \iint_{\Omega \times(0, T)}\left(J_{1, i}+2 J_{2, i}\right)\left(\widetilde{\boldsymbol{m}}^{\infty}(\boldsymbol{x}, t) \wedge \boldsymbol{w}_{i}^{\infty}(\boldsymbol{x}, t)\right) \cdot\left(\boldsymbol{\phi}\left(\boldsymbol{x}, t, z_{i}^{+}\right)-\boldsymbol{\phi}\left(\boldsymbol{x}, t, z_{i}^{-}\right)\right) \mathrm{d} \boldsymbol{x} \mathrm{d} t=0
\end{aligned}
$$

for all $T>0$, for all $\boldsymbol{\phi}$ in $\mathcal{C}^{\infty}(\overline{\Omega \times(0, T)}) \otimes \mathcal{C}_{\#}^{\infty}(Y)$. We choose $\phi(\boldsymbol{x}, t, \cdot)$ having compact support in $\widetilde{I}$. We obtain that $\frac{\partial \widetilde{\boldsymbol{m}}_{1}^{\infty}}{\partial y}(\boldsymbol{x}, t, \cdot)$ is constant on each interval $\widetilde{I}_{i}$. And,

$$
\begin{aligned}
\sum_{i=0}^{p-1} \iint_{Q_{T}} \int_{I_{i}} A_{i}\left(\widetilde { \boldsymbol { m } } ^ { \infty } \wedge \left(\frac{\partial \widetilde{\boldsymbol{m}}^{\infty}}{\partial x_{3}}(\boldsymbol{x}, t)\right.\right. & \left.\left.+\boldsymbol{v}_{i}^{\infty}(\boldsymbol{x}, t)\right)\right) \cdot[\boldsymbol{\phi}(\boldsymbol{x}, t, \cdot)]_{z_{i}^{+}}^{z_{i+1}^{-}} \mathrm{d} \boldsymbol{x} \mathrm{d} t \\
& +\sum_{i=0}^{p-1} \iint_{Q_{T}}\left(J_{1, i}+2 J_{2, i}\right)\left(\widetilde{\boldsymbol{m}}^{\infty}(\boldsymbol{x}, t) \wedge \boldsymbol{w}_{i}^{\infty}(\boldsymbol{x}, t)\right) \cdot[\boldsymbol{\phi}(\boldsymbol{x}, t, \cdot)]_{z_{i}^{-}}^{z_{i}^{+}} \mathrm{d} \boldsymbol{x} \mathrm{d} t=0 .
\end{aligned}
$$

Thus, for every $\boldsymbol{x}, t$ in $\Omega \times \mathbb{R}^{+}$, for any sequences of real numbers $a_{i}, a_{i}^{\prime}$ such that $\sum_{i=0}^{p-1} a_{i}+a_{i}^{\prime}=0$, we have:

$$
\sum_{i=0}^{p-1} \widetilde{\boldsymbol{m}}^{\infty} \wedge\left(a_{i} A_{i}\left(\frac{\partial \widetilde{\boldsymbol{m}}^{\infty}}{\partial x_{3}}(\boldsymbol{x}, t)+\boldsymbol{v}_{i}^{\infty}(\boldsymbol{x}, t)\right)+a_{i}^{\prime}\left(J_{1, i}+2 J_{2, i}\right) \boldsymbol{w}_{i}^{\infty}(\boldsymbol{x}, t)\right)=0 .
$$


There exists a function $\boldsymbol{C}(\boldsymbol{x}, t)$ such that, for every integer $i, 0 \leq i \leq p-1$,

$$
\begin{aligned}
A_{i}\left(\widetilde{\boldsymbol{m}}^{\infty} \wedge \frac{\partial \widetilde{\boldsymbol{m}}^{\infty}}{\partial x_{3}}(\boldsymbol{x}, t)+\boldsymbol{v}_{i}^{\infty}(\boldsymbol{x}, t)\right)=\boldsymbol{C}(\boldsymbol{x}, t), \\
\left(J_{1, i}+2 J_{2, i}\right) \widetilde{\boldsymbol{m}}^{\infty} \wedge \boldsymbol{w}_{i}^{\infty}(\boldsymbol{x}, t)=\boldsymbol{C}(\boldsymbol{x}, t) .
\end{aligned}
$$

As $\left|\boldsymbol{m}^{N}\right|=1$ almost everywhere, for all integers $i$ between 0 and $p-1$,

$$
\begin{gathered}
\widetilde{\boldsymbol{m}}^{\infty}(\boldsymbol{x}, t) \cdot \boldsymbol{v}_{i}^{\infty}(\boldsymbol{x}, t)=0 \text { a.e. in } \Omega \times \mathbb{R}^{+}, \\
\widetilde{\boldsymbol{m}}^{\infty}(\boldsymbol{x}, t) \cdot \boldsymbol{w}_{i}^{\infty}(\boldsymbol{x}, t)=0 \text { a.e. in } \Omega \times \mathbb{R}^{+} .
\end{gathered}
$$

Thus, there exists $\boldsymbol{C}^{\prime}(\boldsymbol{x}, t)$ orthogonal to $\widetilde{\boldsymbol{m}}(\boldsymbol{x}, t)$ almost everywhere, such that

$$
\begin{aligned}
A_{i}\left(\frac{\partial \widetilde{\boldsymbol{m}}^{\infty}}{\partial x_{3}}(\boldsymbol{x}, t)+\boldsymbol{v}_{i}^{\infty}(\boldsymbol{x}, t)\right) & =\boldsymbol{C}^{\prime}(\boldsymbol{x}, t), \\
\left(J_{1, i}+2 J_{2, i}\right) \boldsymbol{w}_{i}^{\infty}(\boldsymbol{x}, t) & =\boldsymbol{C}^{\prime}(\boldsymbol{x}, t) .
\end{aligned}
$$

By Lemma 20, we have:

$$
\boldsymbol{C}^{\prime}(\boldsymbol{x}, t)=\frac{\frac{\partial \widetilde{\boldsymbol{m}}^{\infty}}{\partial x_{3}}(\boldsymbol{x}, t)}{\sum_{i=0}^{p-1}\left(\frac{\bar{\chi}_{i}}{A_{i}}+\frac{1}{L\left(J_{1, i}+2 J_{2, i}\right.}\right)} .
$$

In order to express equation (6.13) with $\widetilde{\boldsymbol{m}}^{\infty}$ as the only unknown, it remains to compute the homogenized demagnetization field term $\boldsymbol{h}^{\infty}$ :

Proposition 22. The term $\mathcal{H}_{d}\left(\chi^{N} \widetilde{\boldsymbol{m}}^{N}\right)$ two-scale converges to

$$
\begin{cases}\bar{\chi} \mathcal{H}_{d}\left(\widetilde{\boldsymbol{m}}^{\infty}\right)-(1-\bar{\chi})\left(\widetilde{\boldsymbol{m}}^{\infty}(\boldsymbol{x}, t) \cdot \boldsymbol{e}_{3}\right) \boldsymbol{e}_{3} & \text { if } y \in \widetilde{I}, \\ \bar{\chi} \mathcal{H}_{d}\left(\widetilde{\boldsymbol{m}}^{\infty}\right)+\bar{\chi}\left(\widetilde{\boldsymbol{m}}^{\infty}(\boldsymbol{x}, t) \cdot \boldsymbol{e}_{3}\right) \boldsymbol{e}_{3} & \text { if } y \in \widetilde{J} .\end{cases}
$$

Proof of the proposition. We do as Hamdache in [9], Proposition 3.7. Let $\varphi^{N}$ be the potential function in $\mathrm{L}^{\infty}\left(0,+\infty ; \mathrm{H}_{\text {loc }}^{1}\right)$ such that $\nabla \varphi^{N}=\mathcal{H}_{d}\left(\chi^{N} \widetilde{\boldsymbol{m}}^{N}\right)$. Thus,

$$
\operatorname{div}\left(\nabla \varphi^{N}+\chi^{N} \widetilde{\boldsymbol{m}}^{N}\right)=0
$$

where $\chi^{N}(\boldsymbol{x})$ is the characteristic function of $\Omega^{N}$. Since $\chi\left(\Omega^{N}\right) \widetilde{\boldsymbol{m}}^{N}$ is bounded in $\mathrm{L}^{\infty}\left(\mathbb{R}^{+} ; \mathrm{L}^{2}\left(\mathbb{R}^{3} ; \mathbb{R}^{3}\right)\right), \varphi^{N}$ is bounded in $\mathrm{L}^{\infty}\left(\mathbb{R}^{+} ; \mathrm{H}_{\text {loc }}^{1}\left(\mathbb{R}^{3}\right)\right)$. Thus, there exist $\varphi^{\infty}$ in $\mathrm{L}^{\infty}\left(\mathbb{R}^{+} ; \mathrm{H}_{\text {loc }}^{1}\left(\mathbb{R}^{3}\right)\right)$ and $\varphi_{1}^{\infty}$ in $\mathrm{L}^{\infty}\left(\mathbb{R}^{+} ; \mathrm{L}_{\text {loc }}^{2}\left(\mathbb{R}^{3} ; \mathrm{H}_{\#}^{1}(Y)\right)\right)$, such that for all $\phi$ in $\mathcal{C}_{c}\left(\mathbb{R}^{3} \times \mathbb{R}^{+}\right) \otimes \mathcal{C}_{\#}^{\infty}(Y)$,

$$
\begin{gathered}
\lim _{N \rightarrow \infty} \iint_{\mathbb{R}^{3} \times \mathbb{R}^{+}} \varphi^{N}(\boldsymbol{x}, t) \phi\left(\boldsymbol{x}, t, \frac{N}{L} x_{3}\right) \mathrm{d} \boldsymbol{x} \mathrm{d} t \\
=\iint_{\mathbb{R}^{3} \times \mathbb{R}^{+}} \varphi^{\infty}(\boldsymbol{x}, t)\left(\int_{Y} \phi(\boldsymbol{x}, t, y) \mathrm{d} y\right) \mathrm{d} \boldsymbol{x} \mathrm{d} t, \\
\lim _{N \rightarrow \infty} \iint_{\mathbb{R}^{3} \times \mathbb{R}^{+}} \frac{\partial \varphi^{\infty}}{\partial x_{3}}(\boldsymbol{x}, t) \phi\left(\boldsymbol{x}, t, \frac{N}{L} x_{3}\right) \mathrm{d} \boldsymbol{x} \mathrm{d} t \\
=\iint_{\mathbb{R}^{3} \times \mathbb{R}^{+}} \int_{Y}\left(\frac{\partial \varphi^{\infty}}{\partial x_{3}}(\boldsymbol{x}, t)+\frac{\partial \varphi_{1}^{\infty}}{\partial y}(\boldsymbol{x}, t, y)\right) \phi(\boldsymbol{x}, t, y) \mathrm{d} y \mathrm{~d} \boldsymbol{x} \mathrm{d} t .
\end{gathered}
$$


But,

$$
\begin{aligned}
& \iint_{\Omega \times \mathbb{R}^{+}} \chi^{N} \widetilde{\boldsymbol{m}}^{N}(\boldsymbol{x}, t) \cdot \nabla_{\boldsymbol{x}} \phi\left(\boldsymbol{x}, t, \frac{N}{L} x_{3}\right) \mathrm{d} \boldsymbol{x} \mathrm{d} t \\
&+\frac{N}{L} \iint_{\Omega \times \mathbb{R}^{+}} \chi^{N}\left(\widetilde{\boldsymbol{m}}^{N}(\boldsymbol{x}, t) \cdot \boldsymbol{e}_{3}\right) \frac{\partial \phi}{\partial y}\left(\boldsymbol{x}, t, \frac{N}{L} x_{3}\right) \mathrm{d} \boldsymbol{x} \mathrm{d} t \\
&=-\iint_{\mathbb{R}^{3} \times \mathbb{R}^{+}} \nabla \varphi^{N} \cdot \nabla_{\boldsymbol{x}} \phi\left(\boldsymbol{x}, t, \frac{N}{L} x_{3}\right) \mathrm{d} \boldsymbol{x} \mathrm{d} t \\
& \quad-\frac{N}{L} \iint_{\mathbb{R}^{3} \times \mathbb{R}^{+}} \frac{\partial \varphi^{N}}{\partial x_{3}} \cdot \frac{\partial \phi}{\partial y}\left(\boldsymbol{x}, t, \frac{N}{L} x_{3}\right) \mathrm{d} \boldsymbol{x} \mathrm{d} t
\end{aligned}
$$

for all $\phi$ in $\mathcal{C}_{c}\left(\mathbb{R}^{3} \times \mathbb{R}^{+}\right) \otimes \mathcal{C}_{\#}^{\infty}(Y)$. Dividing this equality by $N$ and taking the limit, we obtain

$$
\begin{aligned}
\iint_{\Omega \times \mathbb{R}^{+}} \widetilde{\boldsymbol{m}}^{\infty}(\boldsymbol{x}, t) \cdot \boldsymbol{e}_{3}\left(\int_{\tilde{I}} \frac{\partial \phi}{\partial y}(\boldsymbol{x}, t, y) \mathrm{d} y\right) \mathrm{d} \boldsymbol{x} \mathrm{d} t \\
\quad+\iint_{\mathbb{R}^{3} \times \mathbb{R}^{+}} \int_{Y}\left(\frac{\partial \varphi^{\infty}}{\partial x_{3}}(\boldsymbol{x}, t)+\frac{\partial \varphi_{1}^{\infty}}{\partial y}(\boldsymbol{x}, t, y)\right) \cdot \frac{\partial \phi}{\partial y}(\boldsymbol{x}, t, y) \mathrm{d} y \mathrm{~d} \boldsymbol{x} \mathrm{d} t=0
\end{aligned}
$$

Thus, there exists $\Upsilon^{\infty}(\boldsymbol{x}, t)$ such that

$$
\Upsilon^{\infty}(\boldsymbol{x}, t)= \begin{cases}\widetilde{\boldsymbol{m}}^{\infty}(\boldsymbol{x}, t) \cdot \boldsymbol{e}_{3}+\left(\frac{\partial \varphi^{\infty}}{\partial x_{3}}(\boldsymbol{x}, t)+\frac{\partial \varphi_{1}^{\infty}}{\partial y}(\boldsymbol{x}, t, y)\right) & \text { if } y \in \widetilde{I}, \\ \frac{\partial \varphi^{\infty}}{\partial x_{3}}(\boldsymbol{x}, t)+\frac{\partial \varphi_{1}^{\infty}}{\partial y}(\boldsymbol{x}, t, y) & \text { if } y \in \widetilde{J}\end{cases}
$$

Since $\int_{Y} \frac{\partial \varphi_{1}^{\infty}}{\partial y} \mathrm{~d} y=0$

$$
\Upsilon^{\infty}(\boldsymbol{x}, t)=\frac{\partial \varphi^{\infty}}{\partial x_{3}}(\boldsymbol{x}, t)+\bar{\chi} \widetilde{\boldsymbol{m}}^{\infty}(\boldsymbol{x}, t) \cdot \boldsymbol{e}_{3}
$$

Thus, the two-scale limit of $\mathcal{H}_{d}\left(\chi^{N} \widetilde{\boldsymbol{m}}^{N}\right)$ is

$$
\frac{\partial \varphi^{\infty}}{\partial x_{3}}(\boldsymbol{x}, t)+\frac{\partial \varphi_{1}^{\infty}}{\partial y}(\boldsymbol{x}, t, y)= \begin{cases}\frac{\partial \varphi^{\infty}}{\partial x_{3}}(\boldsymbol{x}, t)-(1-\bar{\chi}) \widetilde{\boldsymbol{m}}^{\infty}(\boldsymbol{x}, t) \cdot \boldsymbol{e}_{3} & \text { if } y \in \widetilde{I} \\ \frac{\partial \varphi^{\infty}}{\partial x_{3}}(\boldsymbol{x}, t)+\bar{\chi} \widetilde{\boldsymbol{m}}^{\infty}(\boldsymbol{x}, t) \cdot \boldsymbol{e}_{3} & \text { if } y \in \widetilde{J}\end{cases}
$$

We choose $\phi$ independent of $y$ and take the limit in (6.26), we obtain:

$$
\bar{\chi} \iint_{\Omega \times \mathbb{R}^{+}} \widetilde{\boldsymbol{m}}^{\infty}(\boldsymbol{x}, t) \cdot \nabla_{\boldsymbol{x}} \phi(\boldsymbol{x}, t, y) \mathrm{d} \boldsymbol{x} \mathrm{d} t+\iint_{\mathbb{R}^{3} \times \mathbb{R}^{+}} \nabla \varphi^{\infty} \cdot \nabla \phi \mathrm{d} \boldsymbol{x} \mathrm{d} t=0 .
$$

Thus, $\operatorname{div}\left(\nabla \varphi^{\infty}+\bar{\chi} \widetilde{\boldsymbol{m}}^{\infty}\right)=0$ and $\nabla \varphi^{\infty}(\boldsymbol{x}, t)=\bar{\chi} \mathcal{H}_{d}\left(\widetilde{\boldsymbol{m}}^{\infty}\right)$. Hence, the two-scale limit of $\mathcal{H}_{d}\left(\chi^{N} \widetilde{\boldsymbol{m}}^{N}\right)$ is

$$
\begin{cases}\bar{\chi} \mathcal{H}_{d}\left(\widetilde{\boldsymbol{m}}^{\infty}\right)-(1-\bar{\chi})\left(\widetilde{\boldsymbol{m}}^{\infty}(\boldsymbol{x}, t) \cdot \boldsymbol{e}_{3}\right) \boldsymbol{e}_{3} & \text { if } y \in \widetilde{I} \\ \bar{\chi} \mathcal{H}_{d}\left(\widetilde{\boldsymbol{m}}^{\infty}\right)+\bar{\chi}\left(\widetilde{\boldsymbol{m}}^{\infty}(\boldsymbol{x}, t) \cdot \boldsymbol{e}_{3}\right) \boldsymbol{e}_{3} & \text { if } y \in \widetilde{J}\end{cases}
$$


We merge the results of Propositions 21 and 22 in equation (6.13):

$$
\begin{aligned}
\bar{\chi} \iint_{Q_{T}} \frac{\partial \widetilde{\boldsymbol{m}}^{\infty}}{\partial t} \cdot \boldsymbol{\phi} \mathrm{d} \boldsymbol{x} \mathrm{d} t-\alpha \bar{\chi} \iint_{Q_{T}}\left(\widetilde{\boldsymbol{m}}^{\infty} \wedge \frac{\partial \widetilde{\boldsymbol{m}}^{\infty}}{\partial t}\right) \cdot \boldsymbol{\phi} \mathrm{d} \boldsymbol{x} \mathrm{d} t \\
=\left(1+\alpha^{2}\right) \iint_{Q_{T}} \sum_{i=0}^{p-1} A_{i} \bar{\chi}_{i} \sum_{j=1}^{2}\left(\widetilde{\boldsymbol{m}}^{\infty} \wedge \frac{\partial \widetilde{\boldsymbol{m}}^{\infty}}{\partial x_{j}}\right) \cdot \frac{\partial \boldsymbol{\phi}}{\partial x_{j}} \mathrm{~d} \boldsymbol{x} \mathrm{d} t \\
+\left(1+\alpha^{2}\right) \frac{\bar{\chi}}{\sum_{i=0}^{p-1}\left(\frac{\bar{\chi}_{i}}{A_{i}}+\frac{1}{L\left(J_{1, i}+2 J_{2, i}\right)}\right)} \iint_{Q_{T}}\left(\widetilde{\boldsymbol{m}}^{\infty} \wedge \frac{\partial \widetilde{\boldsymbol{m}}^{\infty}}{\partial x_{3}}\right) \cdot \frac{\partial \boldsymbol{\phi}}{\partial x_{3}} \mathrm{~d} \boldsymbol{x} \mathrm{d} t \\
-\left(1+\alpha^{2}\right) \bar{\chi} \iint_{Q_{T}}\left(\widetilde{\boldsymbol{m}}^{\infty} \wedge\left(\mathcal{H}_{d}\left(\bar{\chi} \widetilde{\boldsymbol{m}}^{\infty}\right)-(1-\bar{\chi})\left(\widetilde{\boldsymbol{m}}^{\infty} \cdot \boldsymbol{e}_{3}\right) \boldsymbol{e}_{3}\right)\right) \cdot \boldsymbol{\phi} \mathrm{d} \boldsymbol{x} \mathrm{d} t \\
\quad+\left(1+\alpha^{2}\right) \iint_{Q_{T}}\left(\widetilde{\boldsymbol{m}}^{\infty} \wedge \sum_{i=0}^{p-1} \bar{\chi}_{i} \mathbf{K}_{i} \widetilde{\boldsymbol{m}}^{\infty}\right) \cdot \boldsymbol{\phi} \mathrm{d} \boldsymbol{x} \mathrm{d} t \\
-\left(1+\alpha^{2}\right) \sum_{i=0}^{p-1} \frac{K_{s, i}^{+}+K_{s, i}^{-}}{L} \iint_{Q_{T}}\left(\boldsymbol{e}_{3} \cdot \widetilde{\boldsymbol{m}}^{\infty}\right)\left(\widetilde{\boldsymbol{m}}^{\infty} \wedge \boldsymbol{e}_{3}\right) \cdot \boldsymbol{\phi} \mathrm{d} \boldsymbol{x} \mathrm{d} t \\
\quad+\left(1+\alpha^{2}\right) \frac{1-\bar{\chi}}{\sum_{i=0}^{p-1}\left(\frac{\bar{\chi}_{i}}{A_{i}}+\frac{1}{L\left(J_{1, i}+2 J_{2, i}\right)}\right)} \iint_{Q_{T}}\left(\widetilde{\boldsymbol{m}}^{\infty} \wedge \frac{\partial \widetilde{\boldsymbol{m}}^{\infty}}{\partial x_{3}}\right) \cdot \frac{\partial \boldsymbol{\phi}}{\partial x_{3}} \mathrm{~d} \boldsymbol{x} \mathrm{d} t
\end{aligned}
$$

for all time $T>0$, for all $\phi$ in $\mathcal{C}^{\infty}\left(\overline{\Omega \times(0, T)} ; \mathbb{R}^{3}\right)$.

\section{Conclusion}

The homogenization of the Landau-Lifshitz equation is non trivial since it is a highly nonlinear partial differential equation. If surface anisotropy dominates, we have obtained satisfactory results up to order 0 . Characterizing the limit up to the first order as $\sqrt{N}\left(\boldsymbol{m}-\left(\boldsymbol{m} \cdot \boldsymbol{e}_{3}\right) \boldsymbol{e}_{3}\right)$ two-scale converges to a first order term is an interesting project for future research.

If surface anisotropy does not dominate the other energy terms, an homogenized equation when the magnitude of super-exchange is inversely proportional to the interlayer distance is obtained. With a weaker super-exchange, no "good" extension operator can be constructed and the convergence of nonlinear terms to their intuitive limits remains uncertain. The homogenization of the Landau-Lifshitz equation in this difficult case would require compactness criteria for strong two-scale convergence analogous to those of Simon [16] for the usual strong convergence.

\section{A. Extensions operators in A MULtilayer}

In this section, we describe one "optimal" extension operator. Let $L>0$. Let $z_{1}, \ldots, z_{N}$ and $z_{1}^{\prime}, \ldots, z_{N}^{\prime}$ be $2 N$ real numbers such that $0<z_{1}, z_{N}^{\prime}<L, z_{i}<z_{i}^{\prime}$ for all $i \in \mathbb{N}, 1 \leq i \leq N$, and $z_{i}^{\prime}<z_{i+1}$ for all integers $i$, $1 \leq i \leq N-1$. We define

$$
\mathcal{I}_{i}=\left(z_{i}, z_{i}^{\prime}\right), \quad \text { for all } i \in \mathbb{N}, \quad 1 \leq i \leq N, \quad \mathcal{I}=\bigcup_{i=1}^{N} \mathcal{I}_{i}, \quad \hat{\mathcal{I}}=\left(z_{1}, z_{N}^{\prime}\right) .
$$

We define $\theta_{i}^{+}$and $\theta_{i}^{-}$for all integers $i, 1 \leq i \leq N-1$ by the formulae:

$$
\theta_{i}^{+}=\frac{z_{i+1}^{\prime}-z_{i+1}}{z_{i+1}-z_{i}^{\prime}}, \quad \theta_{i}^{-}=\frac{z_{i}^{\prime}-z_{i}}{z_{i+1}-z_{i}^{\prime}}
$$




\section{A.1. An "optimal" extension operator}

The following proposition holds:

Proposition 23. Let $B$ be an open set of $\mathbb{R}^{n}, n \in \mathbb{N}, n \geq 1$. Then, there exists an operator $\mathrm{P}$

$$
\mathrm{P}: \quad \mathrm{H}^{1}(B \times \mathcal{I}) \rightarrow \mathrm{H}^{1}(B \times \hat{\mathcal{I}}),
$$

such that for all $u$ belonging to $\mathrm{H}^{1}(B \times \mathcal{I})$ :

$$
\begin{aligned}
\|\mathrm{P}(u)\|_{\mathrm{L}^{\infty}(B \times \hat{\mathcal{I}})} & \leq\|u\|_{\mathrm{L}^{\infty}(B \times \mathcal{I})} \\
\|\mathrm{P}(u)\|_{\mathrm{L}^{2}(B \times \hat{\mathcal{I}})} & \leq(1+2 \alpha)\|u\|_{\mathrm{L}^{2}(B \times \mathcal{I})}, \\
\left\|\partial_{x_{i}} \mathrm{P}(u)\right\|_{\mathrm{L}^{2}(B \times \hat{\mathcal{I}})} & \leq(1+2 \alpha)\left\|\partial_{x_{i}} u\right\|_{\mathrm{L}^{2}(B \times \mathcal{I})}, \quad \text { for } i=1,2 \\
\left\|\frac{\partial \mathrm{P}(u)}{\partial x_{3}}\right\|_{\mathrm{L}^{2}(B \times \hat{\mathcal{I}})} & \leq(1+2 \alpha+2 \beta)\left\|\frac{\partial u}{\partial x_{3}}\right\|_{\mathrm{L}^{2}(B \times \mathcal{I})}+\mu\left(\sum_{i=1}^{N-1}\left\|u\left(\cdot, z_{i+1}\right)-u\left(\cdot, z_{i}^{\prime}\right)\right\|_{\mathrm{L}^{2}(B)}^{2}\right)^{\frac{1}{2}},
\end{aligned}
$$

where $\alpha, \beta, \mu$ are positive real numbers. For example, we choose

$$
\begin{array}{ll}
\alpha=\left(\max _{1 \leq i \leq N-1}\left(\theta_{i}^{+}, \theta_{i}^{-}\right)\right)^{\frac{1}{2}}, & \beta=\left(\min _{1 \leq i \leq N-1}\left(\theta_{i}^{+}, \theta_{i}^{-}\right)\right)^{-\frac{1}{2}}, \\
\mu & =\left(\min _{1 \leq i \leq N-1}\left(z_{i+1}-z_{i}^{\prime}\right)\right)^{-\frac{1}{2}} .
\end{array}
$$

Proof. For all $u$ in $\mathrm{H}^{1}(B \times \mathcal{I})$, we define

$$
\mathrm{P}(u)\left(\hat{\boldsymbol{x}}, x_{3}\right)= \begin{cases}u\left(\hat{\boldsymbol{x}}, x_{3}\right) & \text { if } x_{3} \text { belongs to } \mathcal{I}, \\ \frac{z_{i+1}-x_{3}}{z_{i+1}-z_{i}^{\prime}} u\left(\hat{\boldsymbol{x}}, \theta_{i}^{-}\left(z_{i}^{\prime}-x_{3}\right)+z_{i}^{\prime}\right) & \text { if } x_{3} \text { belongs to }\left(z_{i}^{\prime}, z_{i+1}\right), \\ +\frac{x_{3}-z_{i}^{\prime}}{z_{i+1}-z_{i}^{\prime}} u\left(\hat{v x}, \theta_{i}^{+}\left(z_{i+1}-x_{3}\right)+z_{i+1}\right) & \end{cases}
$$

for all integers $i$ such that $1 \leq i \leq N-1$. The function $\mathrm{P}(u)$ belongs to $\mathrm{H}^{1}(B \times \mathcal{I})$ and the operator $\mathrm{P}$ has the required properties.

\section{A.2. A counter example}

We announced in Section 6.2 that if $\varpi=1$, then we need $\rho=-1$ to have uniform bounds on the $\mathrm{H}^{1}$ norm of the extension. This is true with the operator defined in the previous section but we could always imagine that another extension operator might have better bounds. In this section, we show that it is not possible to improve in a significant manner the operator in every single case. Let's consider a simpler geometry. Let $B$ be a bounded convex open set with a smooth boundary of $\mathbb{R}^{2}$. Let $\theta \in \mathbb{R}^{+}$be such that $0<\theta<\frac{1}{2}$. For all positive integers $N$, we define

$$
\begin{array}{rlrl}
z_{i}^{N,+} & =\frac{i+\theta}{N}, & z_{i}^{N,-}=\frac{i-\theta}{N}, \\
Y^{N}=\bigcup_{i=1}^{N-1}\left(z_{i}^{N,+}, z_{i+1}^{N,-}\right), & Y & =[0,1], \\
\mathcal{O}^{N} & =B \times Y^{N} & \mathcal{O} & =B \times Y .
\end{array}
$$


We also introduce the notations

$$
I^{N}=\bigcup_{\substack{1 \leq i \leq N-1 \\ i \text { odd }}}\left(z_{i}^{N,+}, z_{i+1}^{N,-}\right), \quad J^{N}=\bigcup_{\substack{1 \leq i \leq N-1 \\ i \text { even }}}\left(z_{i}^{N,+}, z_{i+1}^{N,-}\right) .
$$

Then, the following lemma holds:

Lemma 24. Let $\widetilde{u}$ be in $\mathrm{H}^{1}(\mathcal{O})$ such that $\widetilde{u}=u$ on $\mathcal{O}^{N}$. Then,

$$
\left\|\frac{\partial \widetilde{u}}{\partial x_{3}}\right\|_{\mathrm{L}^{2}\left(\mathcal{O} \backslash \mathcal{O}^{N}\right)} \geq \frac{\sqrt{N}}{\sqrt{\theta}}\left(\sum_{i=1}^{N-1}\left\|u\left(\cdot, \cdot, z_{i}^{N,+}\right)-u\left(\cdot, \cdot, z_{i}^{N,-}\right)\right\|_{\mathrm{L}^{2}(B)}^{2}\right)^{\frac{1}{2}} .
$$

Proof. We have

$$
\begin{aligned}
&\left(\sum_{i=1}^{N-1}\left\|u\left(\cdot, \cdot, z_{i}^{N,+}\right)-u\left(\cdot, \cdot, z_{i}^{N,-}\right)\right\|_{\mathrm{L}^{2}(B)}^{2}\right)^{\frac{1}{2}} \\
& \leq\left(\sum_{i=1}^{N-1} \iint_{B}\left|\int_{z_{i}^{N,-}}^{z_{i}^{N,+}} \frac{\partial \widetilde{u}}{\partial x_{3}}\left(x_{1}, x_{2}, x_{3}\right) \mathrm{d} x_{3}\right|^{2} \mathrm{~d} x_{2} \mathrm{~d} x_{1}\right)^{\frac{1}{2}} \\
& \quad \leq \sqrt{\frac{\theta}{N}}\left(\sum_{i=1}^{N-1} \iint_{B} \int_{z_{i}^{N,-}}^{z_{i}^{N,+}}\left|\frac{\partial \widetilde{u}}{\partial x_{3}}\left(x_{1}, x_{2}, x_{3}\right) \mathrm{d} x_{3}\right|^{2} \mathrm{~d} x_{2} \mathrm{~d} x_{1}\right)^{\frac{1}{2}}
\end{aligned}
$$

Let $u^{N}$ be defined by

Then,

$$
u^{N}\left(x_{1}, x_{2}, x_{3}\right)=\frac{1}{\sqrt{N}} \begin{cases}0 & \text { if } x_{3} \text { belongs to } I^{N} \\ 1 & \text { if } x_{3} \text { belongs to } J^{N}\end{cases}
$$

$$
\left(\sum_{i=1}^{N-1}\left\|u^{N}\left(\cdot, \cdot, z_{i}^{N,+}\right)-u^{N}\left(\cdot, \cdot, z_{i}^{N,-}\right)\right\|_{\mathrm{L}^{2}(B)}^{2}\right)^{\frac{1}{2}}=|B|^{\frac{1}{2}}, \quad\left\|u^{N}\right\|_{\mathrm{H}^{1}\left(\mathcal{O}^{N}\right)} \leq \frac{\sqrt{2}}{2} .
$$

According to Lemma 24, if $\widetilde{u}^{N}$ is an extension of $u^{N}$ on $\mathcal{O}$, then

$$
\left\|\widetilde{u}^{N}\right\|_{H^{1}(\mathcal{O})} \geq \frac{\sqrt{N}}{\sqrt{2}}|B|^{\frac{1}{2}} .
$$

Acknowledgements. I am especially grateful to L. Halpern and S. Labbé for their help when working on this subject. I also thank G. Allaire for relevant suggestions.

\section{REFERENCES}

[1] A. Aharoni, Introduction to the theory of ferromagnetism. Oxford Science Publication (1996).

[2] G. Allaire, Homogenization and two-scale convergence. SIAM J. Math. Anal. 23 (1992) 1482-1518.

[3] G. Allaire, A. Damlamian and U. Hornung, Two-scale convergence on periodic surfaces and applications, in Proc. of the International Conference on Mathematical Modelling of Flow through Porous Media, Singapore, May 1995, A. Bourgeat et al. Eds., World Scientific Pub., 15-25.

[4] F. Alouges and A. Soyeur, On global weak solutions for Landau-Lifshitz equations: existence and nonuniqueness. Nonlinear Anal. Theory Methods Appl. 18 (1992) 1071-1084.

[5] W.F. Brown, Micromagnetics. Interscience Publishers (1963).

[6] M.J. Friedman, Mathematical study of the nonlinear singular integral magnetic field equation I. SIAM J. Appl. Math. 39 (1980) 14-20. 
[7] H. Haddar and P. Joly, Homogenized model for a laminar ferromagnetic medium. Proc. Roy. Soc. Edinburgh Sect. A 133, (2003) 567-598.

[8] L. Halpern and S. Labbé, La théorie du micromagnétisme. Modélisation et simulation du comportement des matériaux magnétiques. Matapli 66 (2001) 77-92.

[9] K. Hamdache, Homogenization of layered ferromagnetic media. Preprint 495, CMAP Polytechnique, UMR CNRS 7641, Palaiseau, France (2002).

[10] A. Kirilyuk, J. Ferré, V. Grolier, J. Jamet and D. Renard. Magnetization reversal in ultrathin ferromagetic films with perpendicular anisotropy. J. Magn. Magn. Mater. 171 (1997) 45-63.

[11] M. Labrune and J. Miltat, Wall structure in ferro / antiferromagnetic exchange-coupled bilayers: a numerical micromagnetic approach. J. Magn. Magn. Mater. 151 (1995) 231-245.

[12] L.D. Landau and E.M. Lifshitz, On the theory of the dispersion of magnetic permeability in ferromagnetic bodies. Phys. Z. Sowjetunion 8 (1935) 153-169.

[13] M. Neuss-Radu, Homogenization techniques. Diplomaarbeit, University of Heidelberg (1992).

[14] M. Neuss-Radu, Some extensions of two-scale convergence. C. R. Acad. Sci. Paris Sér. I Math. 322 (1996) 899-904.

[15] K. Santugini-Repiquet, Solutions to the Landau-Lifshitz system with nonhomogeneous Neumann boundary conditions arising from surface anisotropy and super-exchange interactions in a ferromagnetic media. Nonlinear Anal. 65 (2006) 129-158.

[16] J. Simon, Compact sets in the space $\mathrm{L}^{p}(0, T ; B)$. Ann. Mat. Pura Appl. 146 (1987) 66-96.

[17] É. Trémolet de Lacheisserie, editor. Magnétisme: Fondements, Collection Grenoble Sciences, Vol. I, EDP Sciences (2000).

[18] É. Trémolet de Lacheisserie, editor. Magnétisme: Matériaux et applications, Collection Grenoble Sciences, Vol. II, EDP Sciences (2000) 